\title{
A re-examination of Hebbian-covariance rules and spike timing-dependent plasticity in cat visual cortex in vivo
}

\author{
Yves Frégnac ${ }^{1}{ }^{*}$, Marc Pananceau $^{1,2}$, Alice René ${ }^{1}$, Nazyed Huguet ${ }^{1}$, Olivier Marre ${ }^{1}$, Manuel Levy $^{1}$ and \\ Daniel E. Shulz'
}

1 Centre National de la Recherche Scientifique, Unité de Neuroscience, Information et Complexité, Gif-sur-Yvette, France

2 Université Paris-Sud, Orsay, France

Edited by:

Henry Markram, Ecole Polytechnique

Federale de Lausanne, Switzerland

Reviewed by:

Jochen Triesch, Johann Wolfgang

Goethe University, Germany

John Lisman, Brandeis University, USA

Nicolangelo L. lannella, RIKEN Brain

Institute, Japan

*Correspondence:

Yves Frégnac, Centre National de la

Recherche Scientifique, Unité de

Neuroscience, Information et

Complexité, UPR CNRS 3293,

Gif-sur-Yvette F-91198, France.

e-mail:fregnac@unic.cnrs-gif.fr

Spike timing-dependent plasticity (STDP) is considered as an ubiquitous rule for associative plasticity in cortical networks in vitro. However, limited supporting evidence for its functional role has been provided in vivo. In particular, there are very few studies demonstrating the co-occurrence of synaptic efficiency changes and alteration of sensory responses in adult cortex during Hebbian or STDP protocols. We addressed this issue by reviewing and comparing the functional effects of two types of cellular conditioning in cat visual cortex. The first one, referred to as the "covariance" protocol, obeys a generalized Hebbian framework, by imposing, for different stimuli, supervised positive and negative changes in covariance between postsynaptic and presynaptic activity rates. The second protocol, based on intracellular recordings, replicated in vivo variants of the theta-burst paradigm (TBS), proven successful in inducing long-term potentiation in vitro. Since it was shown to impose a precise correlation delay between the electrically activated thalamic input and the TBS-induced postsynaptic spike, this protocol can be seen as a probe of causal ("pre-before-post") STDP. By choosing a thalamic region where the visual field representation was in retinotopic overlap with the intracellularly recorded cortical receptive field as the afferent site for supervised electrical stimulation, this protocol allowed to look for possible correlates between STDP and functional reorganization of the conditioned cortical receptive field. The rate-based "covariance protocol" induced significant and large amplitude changes in receptive field properties, in both kitten and adult V1 cortex. The TBS STDP-like protocol produced in the adult significant changes in the synaptic gain of the electrically activated thalamic pathway, but the statistical significance of the functional correlates was detectable mostly at the population level. Comparison of our observations with the literature leads us to re-examine the experimental status of spike timing-dependent potentiation in adult cortex. We propose the existence of a correlation-based threshold in vivo, limiting the expression of STDP-induced changes outside the critical period, and which accounts for the stability of synaptic weights during sensory cortical processing in the absence of attention or reward-gated supervision.

\section{Keywords: Hebb, intracellular, correlation, potentiation, depression, receptive field, V1, adult plasticity}

\section{INTRODUCTION}

Our understanding of the potential role of associative synaptic plasticity in the malleability of cortical network function during development, perception and learning has up to now been heavily influenced by a single, simple but seminal concept (Hebb, 1949): that the correlational structure of activity patterns between pre- and postsynaptic neurons determines the changes in the transmission efficacy of synaptic connections.

\footnotetext{
Abbreviations: ABS, Artola-Bröcher-Singer plasticity rule; BCM, BienenstockCooper-Munro plasticity rule; LGN, lateral geniculate nucleus; LTD, long-term depression; LTP, long-term potentiation; mPSP, monosynaptic postsynaptic potential; NMDA, $N$-methyl D-aspartate; pPSP, polysynaptic postsynaptic potential; PSTH, post-stimulus time histogram; PSTW, post-stimulus time waveform; RF, receptive field; SDO, sensitivity-direction-orientation polar selectivity analysis; STDP, spike timing-dependent plasticity; $S+$, positive covariance between pre- and postsynaptic activities; S-, negative covariance between pre- and postsynaptic activities; TBS, theta-burst stimulation; TBS_S+, theta-burst stimulation associated with an intracellular current pulse; $\mathrm{V} 1$, primary visual cortex (area 17 in the cat).
}

According to Hebb's rule, the change of the weight from a presynaptic neuron to a postsynaptic neuron depends only on the spiking history of the presynaptic cell and postsynaptic neurons, but does not take into account changes at other neurons "unseen" by the active synapse or other contextual signals. In spite of the fact that Hebb's rule only predicts strengthening of synaptic weights, most theoretical algorithms inspired by Hebb include both associative potentiation and normative depression rules (reviews in Brown et al., 1990; Bi and Poo, 2001; Frégnac, 2002; Gerstner and Kistler, 2002; Brown and Milner, 2003). The Hebbian rule has been the basis of several classical rate-based models applied to unsupervised learning (Oja, 1982; Kohonen, 1989) and developmental and functional epigenesis (Von der Malsburg, 1973; Bienenstock et al., 1982) in cortical networks. Its formalism has been further adapted to follow the timing precision of the spiking process itself (Gerstner et al., 1996; Abbott and Nelson, 2000; van Rossum et al., 2000; Gerstner and Kistler, 2002). 
Although, Hebbian algorithms were formulated as a two-factor rule based on firing rates rather than spike events, their application to the in vivo situation appeared rapidly limited by the presence of on-going activity, hence pre-existing correlations, in the resting state of the network, and by the local nature of the rule, limited to the active synaptic site. In particular, these rules did not take into account other information related to the on-going internal state of the network in which the considered neuron was embedded, or the general stimulus-driven or learning context. The inclusion of an additional control factor can be seen as a form of "meta-plasticity" (plasticity of the induction or expression of plasticity) and allows a permissive graded control of the expression of Hebbian plasticity in primary visual cortex, known to occur during critical periods of development (Bienenstock et al., 1982; Bear et al., 1987). It accounts for the observed gating of cortical plasticity, through the permissive action of noradrenergic and dopaminergic "print now" neuromodulatory signals (Crow, 1968; Kety, 1970) and oculomotor proprioceptive reafference (Frégnac, 1987). It also complies to the synaptic tagging hypothesis, where prior activity at a synapse changes its ulterior susceptibility to undergo synaptic potentiation (Frey and Morris, 1997). Other versions of three-factor rules were later introduced, which attributed a specific gating role to diffusible brain-derived neurotrophic factors in hippocampal longterm potentiation (LTP) and to nitric oxide in cerebellar long-term depression (LTD) (Crepel, 1998). Similar three-term rules have been generalized to incorporate the behavioral context of classical conditioning in a Hebbian framework (Klopf, 1988).

The more advanced variants of Hebb's rule share the same general equation, where the change of synaptic efficacy with respect to time is equal to the product of three variables: one is contextual, and linked to state-dependent control and learning efficiency, and the two remaining terms are linked respectively to presynaptic and postsynaptic activity (reviews in Frégnac and Shulz, 1994; Frégnac, 2002). The so-called "covariance hypothesis" introduced by Sejnowski (1977) and applied in visual cortex by Bienenstock et al. (1982) uses a multiplicative scalar controlling learning efficiency and replaces the pre- and postsynaptic terms by the departure of instantaneous pre- and postsynaptic activities from their (or a non-linear function of their) respective average values over a certain time window. Since the multiplication of the two activitydependent terms is mathematically equivalent to a covariance product, the rule obeys a "sign rule" and predicts potentiation of synaptic efficacy when pre- and post-activities increase phasically together (positive covariance) and depression when one term increases while the other decreases (negative covariance). The theoretical sophistication of the BCM rule is that it includes a local postsynaptic "floating plasticity threshold," which avoids saturation or cancellation of synaptic weights and results in self-normalization (see Frégnac, 2002 for a more extensive review). Additional processes, such as synaptic scaling and synaptic redistribution have been since proposed to account for a more global homeostasis of the mean network activity irrespectively of distributed associative synaptic changes (Abbott and Nelson, 2000).

The validity of these theoretical learning rules has been investigated experimentally in Hebbian supervised paradigms where the first contextual term is set arbitrarily in the permissive state: irrespectively of the internal state of the preparation, an external supervisor (most of the time, the experimenter!) imposes an artificial correlational state between pre- and postsynaptic neurons. Experiments, including those from our laboratory, show classically that forced coincident activity induces LTP of synaptic efficiency, whereas noncoincident activity either evokes LTD or no change (Kelso et al., 1986; Frégnac et al., 1988; Reiter and Stryker, 1988; Bear et al., 1990; Bear and Malenka, 1994; review in Frégnac, 2002; Figure 1). When first described, the observed plasticity curves (change in synaptic efficiency vs post- and presynaptic delay) were found to be symmetric in time, i.e., no strict temporal ordering was required between the onset of pre- and postsynaptic activation. The temporal contiguity requirement of Hebbian potentiation in sensory neocortex, motor cortex and hippocampus was first estimated in the $\pm 50 \mathrm{~ms}$ range, both in vivo (Baranyi and Feher, 1981) and in vitro (Wigström and Gustafsson, 1985; Frégnac et al., 1994a; Harsanyi and Friedlander, 1997); but see Levy and Steward (1983) and Levy (1985).

In the past 15 years, refined work using dual patch recordings in vitro in silent networks demonstrated an even tighter temporal contingency rule (10 ms range), termed "spike timing-dependent plasticity" and the decisive importance of the temporal order between the test postsynaptic potentials (PSP) and the back propagating postsynaptic spike in deciding whether potentiation or depression occurs (Markram et al., 1997): if the postsynaptic cell fires an action potential a few milliseconds after the presynaptic cell, in such a way as to reproduce a causal pre $\rightarrow$ post relation, LTP is induced, whereas the opposite temporal order results in LTD (Debanne et al., 1997; Markram et al., 1997; Feldman, 2000; Bi and Poo, 2001; Sjöström and Nelson, 2002). Synaptic plasticity, however, was further shown to be also determined by additional non-Hebbian factors, such as the number of postsynaptic spikes in a burst (Sjöström et al., 2001; Froemke and Dan, 2002; Froemke et al., 2005b), postsynaptic depolarization (Sjöström et al., 2001, 2004; Sjöström and Häusser, 2006), and neuromodulation (Kasamatsu et al., 1985; Bear and Singer, 1986; Seol et al., 2007; Pawlak and Kerr, 2008). The outcome of the pairing was shown to depend also on the distance of the synapse from the soma (Froemke et al., 2005a; Letzkus et al., 2006; Sjöström and Häusser, 2006), suggesting the further participation of intrinsic conductance distributions in the dendrites and efficiency of backpropagation of the postsynaptic spike. The spatial gradient of synaptic change along the dendrite results in part from the attenuation of the back propagating action potentials during high frequency trains of action potentials. Dendritic depolarization can boost backpropagation of action potentials and switch plasticity between LTD and LTP at distal dendrites (Sjöström and Häusser, 2006). The action potential attenuation can be persistently counteracted by a long-lasting increase in neuronal intrinsic excitability requiring an elevation of the postsynaptic calcium concentration and the activation of CaMKII (Tsubokawa et al., 2000). This last effect may be highly dependent on the on-going level of inhibition as shown in other sensory systems (Van den Burg et al., 2007). Thus, propagation of action potential back to the dendrite depends on the recent activity of the neuron and its long-term modulation may play a role in the subsequent induction of associative synaptic plasticity.

Over the last 20 years, a large variety of afferent stimulation protocols (Figure 1A) have been used to control both (directly) presynaptic and (indirectly) postsynaptic states and induce LTP and 


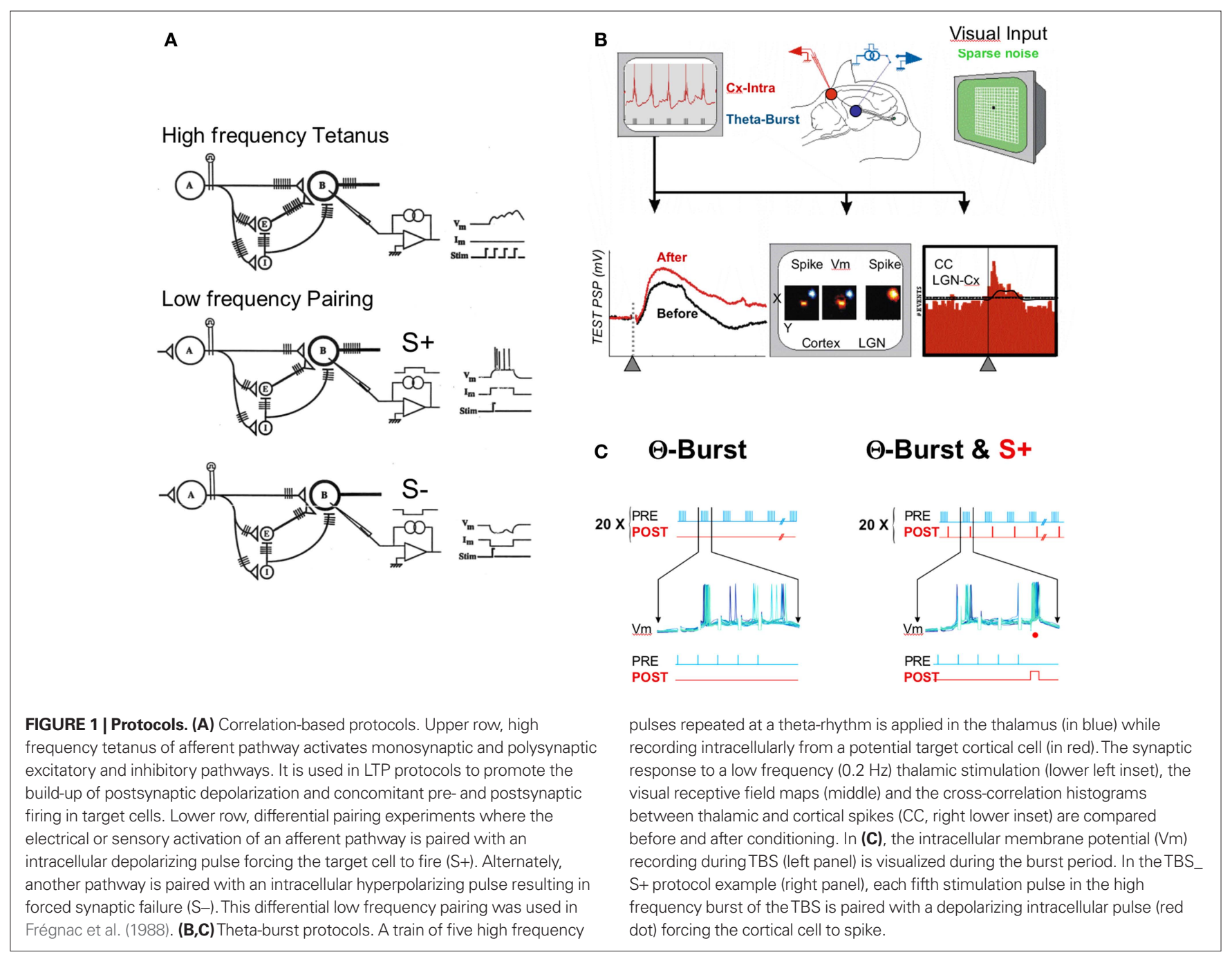

LTD in hippocampal (Dudek and Bear, 1992; Mulkey and Malenka, 1992; Bear and Malenka, 1994; Malenka, 1994) and neocortical slices (Dudek and Bear, 1993; Kirkwood et al., 1993; Kirkwood and Bear, 1994). Unlike in Hebbian supervised paradigms, these protocols did not explicitly require an exogenous control of the postsynaptic discharge pattern. Nevertheless, it is generally admitted that most of their effects can be explained on the basis of the induced correlation between pre- and postsynaptic activities, hence by spike timing-dependent plasticity (STDP) or Hebbian-like processes. Low frequency presynaptic stimulation trains $(1 \mathrm{~Hz}, 900$ pulses) induce LTD, whereas presynaptic theta-burst stimulation [a high frequency $(100 \mathrm{~Hz})$ burst volley repeated at $5-7 \mathrm{~Hz}$ ] induces LTP. The efficiency of these protocols in visual cortex has been reported to be age-dependent when the afferent volley originates from the white matter, and to be strongest at the peak of the critical period in kittens (Kirkwood et al., 1993). A different susceptibility period has been found in supragranular layers: NMDA-receptor activation dependent LTP can be still promoted in adult cortex if the strong inhibitory influence originating from layer IV, and normally elicited by thalamic stimulation, is bypassed pharmacologically (Artola and Singer, 1987) or if the afferent volley is applied directly in the superficial layers (Bear et al., 1992; Kirkwood et al., 1995). Age-dependency regulation is less obvious for LTD induction (but see Dudek and Friedlander, 1996), and strong layer variations have been also observed, with a diversity of molecular pathways involved (dominated by NMDA-receptor activation in layer 2-3 and mGluR in layer 6) and an absence of effect in layer 4 (Rao and Daw, 2004).

On the whole, most of the evidence gathered in vitro suggests that theta-burst patterned stimulation induces a robust developmental form of LTP of thalamo-cortical synapses, in particular in kitten and young rodent visual cortex. This may account for the functional epigenetic changes occurring during the critical period of ocular dominance and orientation preference (Kirkwood et al., 1996; review in Frégnac and Imbert, 1984). The apparent down-regulation of susceptibility of layer IV to express LTP has been replicated in the somatosensory cortex (Crair and Malenka, 1995), which strengthens the parallel drawn between LTP and the critical period of sensitivity to sensory deprivation (review in Foeller and Feldman, 2004).

In spite of these data and the success of STDP as a phenomenological rule accounting for associative plasticity in vitro, limited support for a functional role of LTP has been provided in vivo 
(see Discussion for a more extensive review). In particular, there is very little experimental data exploring co-evolution of synaptic plasticity and changes in sensory responses during Hebbian or STDP protocols, particularly in adult cortex. An example of such an approach can be found in the work of Heynen, Bear and colleagues, trying to relate monocular deprivation, LTD and LTP to bidirectional modifications of visual acuity (Heynen and Bear, 2001; Iny et al., 2006).

The present paper addresses this issue, by reviewing and comparing two series of attempts to modify synaptic efficacy and functional responses in single neurons recorded in kitten and cat visual cortices:

- The first type of protocol used a Hebbian framework to implement, through iontophoretic or intracellular means, supervised positive and negative changes in covariance between postsynaptic and presynaptic activities during the time of recording of the same cell (Figure 1A). The main findings of this already published work are summarized here, since they still constitute the largest functional changes reported so far in a single visual cortical neuron (Frégnac et al., 1992, 1988; Shulz and Frégnac, 1992; Debanne et al., 1998; Frégnac and Shulz, 1999): the alternate imposition, for the same cell, of "high" rates of responses for a given input feature and "low" rates for another input leads to long-lasting changes in sensory responsiveness which favors the response for the positively reinforced feature. The reported effects constitute cellular analogs of functional epigenesis and provide the earliest demonstrations of Hebbian-induced changes in adult cortex. In addition to the forms of associative plasticity predicted by the Hebbian rule and its pseudo-Hebbian correlates (Hebb, 1949; Stent, 1973), these experiments confirm some specific predictions of the covariance hypothesis (Bienenstock et al., 1982). In particular, they outline a form of homosynaptic depression, when presynaptic activity is associated with repetitive failure in synaptic transmission (Reiter and Stryker, 1988; Blais et al., 1999), hence a form of plasticity which requires only a subthreshold postsynaptic change (and no spike).

- The second type of protocol, used in a group of new unpublished intracellular experiments, replicates in vivo variants of the theta-burst paradigm. The rationale of these experiments was twofold: (1) to apply electrical stimulation protocols (theta-burst stimulation, TBS), proven to be successful in inducing LTP in vitro, in order to produce a change in the cortical synaptic response to a test thalamic pathway, and (2) to measure the functional consequence of this artificial activity control on target cortical properties, assessed with visual stimuli. With these two purposes in mind, the electrical test stimulus and the high frequency stimulation burst (TBS used for conditioning) were applied in a thalamic region where the visual field representation was in retinotopic proximity or overlapped with the intracellularly recorded cortical receptive field. Since TBS was shown to improve pre-post synaptic correlation in most of the recorded cells without changing their mean activity, the novelty of this protocol was to provide a probe for functional changes caused by causal STDP mechanisms ("pre-before-post") in adult cat cortex in vivo (Figures 1B,C).
In addition, since each theta-burst input is composed by several presynaptic shocks, and thus creates multiple spike delay interactions within a burst, we superimposed, in certain cells, Hebbian supervised pairings added at a fixed intra-burst phase to the theta-burst (Figure 1C, right panel). These additional experiments show new evidence in vivo of how the supervised reconfiguration of the precise postsynaptic spiking pattern alters in a reversible way the primary effect of high frequency bursts to the cortex.

The Section "Discussion" will compare the various instances of experimental evidence of Hebbian-like or STDP-like correlates of functional plasticity in visual cortex in vivo and re-examine the status of spike timing-dependent LTP in adult cortex.

\section{MATERIALS AND METHODS}

\section{ANIMAL PREPARATION AND RECORDING TECHNIOUES}

Electrophysiological extracellular and intracellular recordings were made in the primary visual cortex of anesthetized and paralyzed kittens and cats, according to the American Physiological Society's Guiding Principles in the Care and Use of Animals. Animals used in these experiments have been bred in the Central CNRS Animal Care facilities at Gif-sur-Yvette. In brief, animals were anesthetized with an intra-muscular injection of alfaxalone/alphadolone (Saffan ${ }^{\circledast}$, Schering-Plough, $13.5 \mathrm{mg} \mathrm{kg}^{-1}$ ), a catheter was inserted into the femoral vein for infusion of anesthetic (alfaxalone/alphadolone, flow rate: $2.6 \mathrm{mg} \mathrm{kg}^{-1} \mathrm{~h}^{-1}$ ) supplemented with isotonic saline and glucose during the remainder of the experiment. After endotracheal cannulation, the animal was positioned in a stereotaxic HorsleyClarke frame. Pancuronium bromide (Pavulon ${ }^{\circledR}$, Organon, flow rate: $0.2 \mathrm{mg} \mathrm{kg}^{-1} \mathrm{~h}^{-1}$ ) was added to the perfusion to prevent eye movements. The animal was artificially ventilated at a rate adjusted to maintain end-tidal $\mathrm{CO}_{2}$ between 3.5 and $4.2 \%$. Body temperature was kept at $38.5^{\circ}$ using a feedback-controlled heating pad. EKG and EEG were monitored continuously to control the proper level of anesthesia through-out the experiment. Ocular application of both atropine 1\% (Europhta) and phenylephrine clorhydrate 5\% (Néosynéphrine ${ }^{\circledast}$, Europhta) was used to dilate the pupils, block accommodation, and retract the nictitating membranes. Eyes were refracted, fitted with the appropriate corrective lenses and focused on the monitor screen set at $57 \mathrm{~cm}$ from the eyes. Small craniotomies (less than $4 \mathrm{~mm}$ diameter) were made over the dorso lateral geniculate nucleus (LGN) (see section below) and the primary visual cortex. The stability of recording was improved by cementing (GC Reline, GC America Inc.) the skull to additional fixation bars and a small recording chamber was fixed such as to enclose the cranial openings. After dura incision and electrode placement, the holes were filled with agar, heavy mineral oil, or a silicone grease (Kwick-Cast, World Precision Instrument) to seal the recording chamber and protect the underlying cortex from drying.

\section{LGN recording and stimulation}

In all theta-burst experiments, a tungsten microelectrode (2.5-4 M $\Omega$, Frederick Haer) was inserted into the LGN, ipsilateral to the cortical recording site. The electrode tip was positioned in LGN layer A representing the central visual field (stereotaxic Horsley-Clarke coordinates $A=5-6 ; L=8-9 ; p=3-4$ ) at a depth 
of 11-12 mm from the pial surface. The final electrode position was typically adjusted within $100-200 \mu \mathrm{m}$ from the point at which the first contralateral visual responses where encountered. The LGN multi-unit signal was amplified, filtered $(300 \mathrm{~Hz}-10 \mathrm{kHz})$ and sampled (at $8 \mathrm{kHz}$ ) for further off-line spike discrimination. LGN units were typically characterized by a small monocular receptive field (RF) and their ability to follow high temporal frequency stimulation. The recording LGN electrode was also used as a stimulating electrode through which constant current, negative pulses of $0.2 \mathrm{~ms}$ duration were applied at $0.1 \mathrm{~Hz}$ (except for theta-burst). The test LGN stimulation intensities ranged from 40 to $360 \mu \mathrm{A}$, as required to reliably evoke PSP in the simultaneously intracellularly recorded cortical cell. Short and fixed latency responses following $100 \mathrm{~Hz}$ train stimulation were considered as monosynaptic.

\section{Intracellular cortical recordings}

Intracellular recordings of cortical cells were obtained using 60-90 M $\Omega$ sharp electrodes pulled from $1.5 \mathrm{~mm}$ borosilicate glass capillaries (WPI) and filled with $2 \mathrm{M}$ potassium methyl-sulfate (containing $4 \mathrm{mM}$ potassium chloride to avoid tip polarization). The microelectrode was positioned around the retinotopic representation of the area centralis $(p=1.5-2.5 ; L=2-4)$ (Albus, 1975; Tusa et al., 1978), and adjusted when possible to obtain some spatial overlap between the thalamic and the cortical receptive fields. Electrode track penetration started along a latero-medial axis, from the area 17-18 border to the depth of the medial area 17 bank (ranging from 680 to $4150 \mu \mathrm{m}$ ). Intracellular postsynaptic potentials were recorded in current-clamp bridge mode with an Axoclamp-2B amplifier (Axon instruments) and digitized at $8 \mathrm{kHz}$ after adequate low-pass filtering. The EEG was recorded over of the homotopic contralateral cortex of the intracellular recording site. All electrophysiological signals were amplified and filtered in parallel with a CyberAmp 380 (Axon instruments), fed to an A/D interface (DIGIDATA 1200, Axon instruments) port and were further processed using a custommade analysis program (Elphy ${ }^{\mathrm{TM}}$, Sadoc CNRS-UNIC) running on a PC computer.

\section{PLASTICITY PROTOCOLS \\ Covariance-based}

The rationale that was applied to implement the covariance plasticity rule is summarized in Figure 1A. Opposite changes were imposed in the temporal correlation between two test sets of synaptic inputs on the one hand, and the output signal of the cell on the other hand. An external supervisor imposed the cell's rate of firing for a given sensory input (usually a "non-preferred" feature) at a "high" level ( $\mathrm{S}+$ pairing), and, in alternate trials, blocks the cell's response to another distinct (usually "preferred") input (S- pairing). The control of postsynaptic activity was imposed in two ways: for extracellular pairings (electrodes filled with $\mathrm{KCl} 3 \mathrm{M}$, 10-20 M $\Omega$ ), the recordings were juxtacellular (spikes of several $\mathrm{mV}$ and same polarity as intracellular), which allowed the application of small intensity iontophoretic currents (less than $\pm 10 \mathrm{nA}$ ) and recording of the cell's activity even during pairing (see also Andrew and Fagan, 1990). For intracellular pairings (electrodes filled with $\mathrm{KCH}_{3} \mathrm{SO}_{4} 2 \mathrm{M}, 50-100 \mathrm{M} \Omega$ ), a brief pulse of depolarizing or hyperpolarizing current (less than $\pm 3 \mathrm{nA}$ for 50-200 ms) was applied through the intracellular electrode (KMs, 50-70 M $\Omega$ ) and synchronized with the stimulus features according to the stimulation protocol.

\section{STDP-based}

Theta-burst stimulation was applied through the thalamic stimulation electrode (Figure 1B). A TBS train was defined by 10 bursts of 5 pulses at $100 \mathrm{~Hz}$, each burst repeated at a theta frequency $(5 \mathrm{~Hz})$. A conditioning sequence was composed of 25 TBS trains, repeated at every $10 \mathrm{~s}$. Stimulus pulse intensity was set to the test level used to trigger the control PSP. In addition to this protocol and for a restricted number of cells, we also imposed supervised postsynaptic firing at a specific temporal phase during each high frequency burst (TBS_S+ in Figure 1C). This was achieved by injecting brief (4-6 ms) intracellular current pulses (0.5-1.0 nA), while keeping the temporal relation between the current pulse and the high frequency volley constant. Depending on the pairing, the postsynaptic firing was generally imposed for the first or the fifth presynaptic event of the LGN burst.

\section{ANALYSIS OF ELECTRICALLY EVOKED SYNAPTIC RESPONSES}

Measurements of the latency, the initial slope, the time and peak of the maximum response and the integral of the depolarizing component of the PSP relative to the pre-stimulus baseline at each trial were used to quantify synaptic modifications. Fifty to 100 successive thalamo-cortical PSPs triggered at $0.2 \mathrm{~Hz}$ by the LGN stimulation were recorded before and after the TBS application and the level of significance of the changes was assessed by using both parametric (Student $t$-test, $p<0.001$ ) and non-parametric tests (Kolmogorov-Smirnov, $p<0.01$ ). We also partitioned the integral PSP changes in amplitude range blocks by $20 \%$ steps compared to the initial test response.

\section{VISUAL STIMULATION AND ANALYSIS OF RF CHANGES}

Once the RF was localized using a semi-automated search with a scalable drifting bar, the ocular dominance and orientation preference of visual responses were qualitatively determined. Stimulation was maintained through the dominant eye for the remaining part of the experiment. The spatio-temporal structures of the cortical (intracellular) and thalamic (multi-unit) receptive fields were mapped with sparse noise [ $\operatorname{dark}\left(1 \mathrm{~cd} \mathrm{~m}^{-2}\right)$ and bright $\left(25 \mathrm{~cd} \mathrm{~m}^{-2}\right)$ pixels, with a mean screen luminance $\left.\left(13 \mathrm{~cd} \mathrm{~m}^{-2}\right)\right]$. Depending on the cells and the thalamo-cortical RF arrangement, the chosen size of each pixel ranged from $0.2^{\circ}$ to $0.7^{\circ}$ (mean: $0.5^{\circ}$ ) to cover an explored region of $8-20^{\circ}$. ON and OFF durations were usually set at 26.7 or $53.4 \mathrm{~ms}$ (corresponding to $4-8$ consecutive frames for a $150-\mathrm{Hz}$ refresh rate monitor).

The forward correlations with visual stimulation of the subthreshold (membrane potential) response of the cortical cell and the supra-threshold (action potentials) responses of the simultaneously recorded thalamic and cortical cells were computed for each position and contrast of the stimulus. Post-stimulus time histograms (PSTH) of the visually evoked discharges and the post-stimulus time waveform (PSTW) of the subthreshold responses were then integrated over a $50 \mathrm{~ms}$ duration sliding window (in $1 \mathrm{~ms}$ steps). $\mathrm{X}$-Y and $\mathrm{X}$-t receptive field maps were then expressed as a $Z$-score relative to the on-going activity prior to the response onset. The 
optimal receptive field map was defined as the map taken at the post-stimulus latency at which a significant response (given by the $Z$-score value compared to pre-stimulus condition, $p<0.05$ ) was observed for the largest number of pixel positions. The optimal RF maps taken before and after the TBS application at the same latencies were compared to quantify the functional impact of the thalamo-cortical synaptic plasticity on the RF structure.

Modification of the spatial RF was quantified using a polar analysis carried out on the optimal X-Y maps: the cortical visual response profile was integrated on a radial partition of the RF space in 24 sectors of $15^{\circ}$ centered on the initial cortical RF center before TBS (Figure 8, left cartoon). The bisector of the first sector was aligned for each cell with the reference axis defined by the alignment of the cortical and thalamic RF centers, such that $0^{\circ}$ designated a RF shift toward the LGN-RF center. This polar representation made it possible to apply quantification measures which have been used classically for the study of orientation and direction tuning modifications (Wörgötter and Eysel, 1987). An sensitivity-direction-orientation (SDO) analysis was used to measure the polar and directional selectivity of the change in the sector-based distribution produced by the TBS pairing. This calculus is based on the assumption that the radial distribution of RF changes, pooled over all conditioned cells, can be approximated by an angular $(\alpha)$ cosine function of the form $R(\alpha)=A o+\Sigma_{j}[A j \cos (j \alpha)]$, with the summation index $\mathrm{j}$ taking a value of «1» for directional and « $2 »$ for orientational tuning, corresponding to a truncated expansion of the Fourier decomposition limited to the first two harmonics. The phase and gain of the first-order and second-order components are measured with a Fast Fourier Transform. The SDO analysis allows the extraction of an index of anisotropy (IA) of the spatial RF change (as the gain of the 1st order component of the decomposition, equivalent to the strength (D) in Wörgötter and Eysel annotation) and the Direction of Anisotropy $(\Theta)$, i.e., the most likely direction of the spatial change (as the phase of the 1st order component, equivalent to the preferred direction (PD) in Wörgötter and Eysel annotation): thus IA and $\Theta$ give respectively the norm and the angle of a vector representing the average weighted shift in RF anisotropy.

\section{RESULTS \\ PROTOCOL 1: SUPERVISION OF COVARIANCE BETWEEN PRE- AND POSTSYNAPTIC ACTIVITIES}

A classical approach used to demonstrate the functional implication of Hebbian-like mechanisms in vivo relies on the study of various forms of visual cortical plasticity induced by manipulations of environmental features, during development and in adulthood (review in Frégnac and Imbert, 1984). The plasticity protocols reviewed here focus on the consequences of Hebbian rules at the individual cell level. Rather than submitting the entire cortical network to an environmental "surgery" of the whole visual field (global clamp of cortex input), cellular analogs of learning restrict the extent to which cortical activity is modulated to the immediate environment of the recorded cell (local perturbation mode). With this approach, the experimenter controls the postsynaptic firing of the recorded cell and imposes a supervision signal which will simulate locally the functional effects of anomalous visual experience during critical periods of development, whereas the majority of the "unseen" units in the network remain unaffected. Two techniques of postsynaptic activity supervision were achieved: (1) by iontophoretic pulses applied through the juxtacellular $\mathrm{KCl}$ recording electrode or (2) by current pulses using intracellular techniques (see Materials and Methods). The first method was better suited to control the firing rate whereas the second one imposed precisely the spike timing of the conditioned cells.

In collaboration with Elie Bienenstock, Simon Thorpe, Dominique Debanne and Attila Baranyi, we (Yves Frégnac and Daniel E. Shulz) developed some 25 years ago a series of electrophysiological supervised Hebbian paradigms (Frégnac et al., 1988, 1992, 1994a,b; Shulz and Frégnac, 1992; Shulz et al., 1993; Debanne et al., 1995, 1998; Frégnac and Shulz, 1999). These sets of experiments were devised to quantify the functional impact of supervised control of covariance between pre- and postsynaptic activity and compare the observed effects with the predictions of theoretical models, specifically the so-called BCM rule introduced by Elie Bienenstock and Leon Cooper's group in their seminal paper (Bienenstock et al., 1982).

The differential pairing protocols presented in Figure 2 have been considered as cellular analogs of functional epigenesis of mammalian V1 since they reproduce functional changes occurring without supervision in freely behaving animals, during development or following early manipulation of the visual environment, for instance an orientation-biased environment (Figure 2A), monocular deprivation (Figure 2B), optically induced interocular orientation disparity and rearing restricted to a fixed phase and spatial frequency (data not shown; Shulz and Frégnac, 1992). Figures 2C,D illustrate the effects of covariance-based pairing protocols on the spatial ON-OFF (or Simple/Complex) organization of visual cortical receptive fields. Surprisingly, at least in the eyes of the reviewers when this work was submitted, the probability of inducing functional changes was reported to be comparable in the kitten during the critical period and in older kittens and adults, suggesting that plasticity might extend well beyond the classical critical period in the presence of an external supervision signal provided by the experimenter, attention or behavioral reward (Frégnac et al., 1988). Since the local supervised learning procedures, applied at the cellular level, imposed an external control of the evoked discharge (through current injection and potassium iontophoresis or field effects), these findings suggest that this type of supervision might bypass systemic homeostatic mechanisms which normally block the expression of plasticity in the mature brain. However, the largest effects were induced in the youngest animals at the peak of the critical period. The major findings are summarized below.

\section{Orientation selectivity plasticity}

Early studies on the effects of visual exposure restricted to a fixed orientation (Blakemore and Cooper, 1970; Hirsch and Spinelli, 1970) showed the induction of a significant bias in the cortical representation in favor of the orientation to which kittens had been exposed. Two different interpretations were historically proposed, calling for either selective (the "functional verification" hypothesis) or instructive ("tabula rasa" alternative) mechanisms. However, in view of the inherent limitations of analysis based on the comparison of populations of neurons recorded extracellularly in different animals, no definitive answer could 


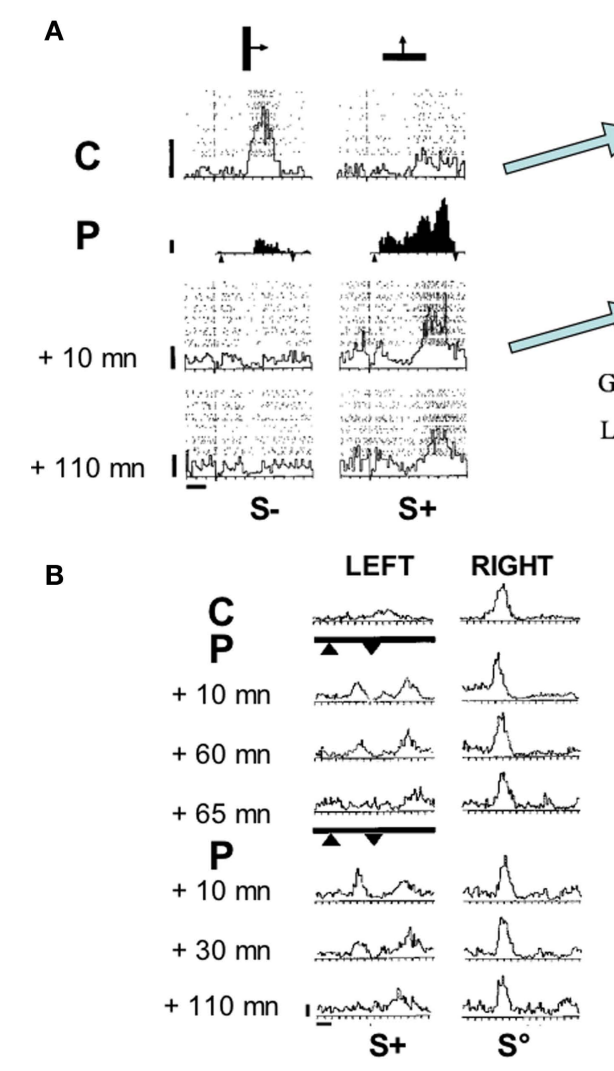

FIGURE 2 | Functional impact of the covariance-based algorithm in kitten V1. (A) Change in orientation preference (adapted from Frégnac et al., 1992): Cell recorded in a visually deprived kitten at the peak of the critical period. Left panel: PSTHs represent visual responses to a moving bar for two different orientations (40 runs each). Superimposed dot displays represent spiking responses for each individual trial. Left column, responses for the initially preferred stimulus (vertical orientation). Right, initially non-preferred stimulus (horizontal orientation). From top to bottom, evolution of the relative preference as a function of time, before (C: control), during differential pairing (P) and at two delays following pairing. During pairing ( $\mathrm{P}$, filled histograms, 60 associations), a positive current pulse ( $+3 \mathrm{nA})$ was applied during the sweep of the horizontal bar across the discharge field (arrowheads, $\mathrm{S}+$ ), and interleaved with a negative current pulse (-7nA) when the vertical bar was presented (arrowheads, S-). The visual response became respectively potentiated for the $\mathrm{S}+$ stimulus and depressed for the $\mathrm{S}$ - orientation (+10 $\mathrm{min})$. The effects were still present $110 \mathrm{~min}$ after pairing. Calibration bars: vertical $5 \mathrm{ap} \mathrm{s}^{-1}$, horizontal $1 \mathrm{~s}$ and $1.5^{\circ}$. Right panel: Polar orientation tuning curves were established for the same cell before (Control) and after pairing (+10 min). The mean spontaneous activity level is shown by the stippled area. The orientations used during pairing are indicated by $\mathrm{S}+/ \mathrm{S}-$ symbols. The lower graph represents the differences between the normalized tuning curves before and after pairing (folded on a $180^{\circ}$ scale) expressed as gains and losses as a function of the orientation of the stimulus (calibration: $\pm 20 \%$ ). Following pairing, the cell changed its orientation preference by $90^{\circ}$, and became tuned to the positively-reinforced orientation and direction. (B) Ocular dominance change (adapted from Shulz and Frégnac, 1992). Cell recorded in a 4.5-week-old normally reared kitten. PSTHs represent visual responses to stimulation of the left (left column) and the right (right column) eyes, before and after two pairings (thick lines). The increase of the visual response to the left eye $(+40 \%)$, imposed during the first pairing ( $9 \mathrm{~S}+$ pairings)
D
S-

$\mathrm{S}+$

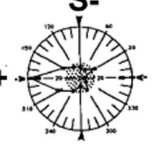

Loss $]-1$

s+ S-

C

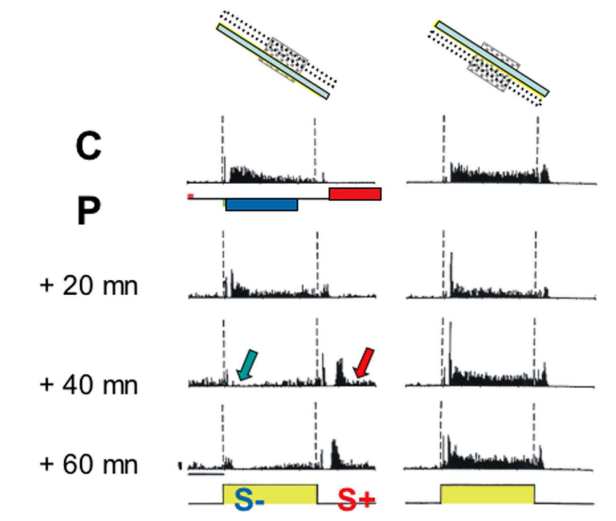

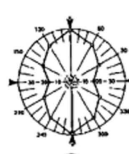

was retained for $60 \mathrm{~min}$. After extinction (+65 min), this effect was reinstated by a second pairing (imposing a $90 \%$ increase in firing during $24 \mathrm{~S}+$ trials), which was retained for $110 \mathrm{~min}$. The response to stimulation through the unpaired $\left(\mathrm{S}^{\circ}\right)$ right eye was unchanged. Calibration: vertical $10 \mathrm{ap} \mathrm{s}^{-1}$, horizontal $1 \mathrm{~s}$ and $1.5^{\circ}$. (C) Change of the ON/OFF balance (adapted from Debanne et al., 1998). Cell recorded in a 6-week-old kitten. PSTHs represent the cell's response to the presentation (ON) and extinction (OFF) of an optimally oriented bar in a fixed position of the RF (cartoon). Before pairing (C, control), a tonic "ON" response and a more transient "OFF" response were observed uniformly across the RF. The pairing procedure ( $\mathrm{P}$, data not shown) consisted of 50 associations of a negative current pulse (-3nA, 2120 ms duration) with the onset of the light bar and a positive current pulse of similar duration $(+3.2 \mathrm{nA})$ following the offset of the same stimulus with a constant delay of $500 \mathrm{~ms}$. A progressive change developed over 40 min after pairing, resulting in a significant depression of the "ON" response $(p<0.0005)$, whereas a late "OFF" response appeared de novo in the paired position. The latency of the new response precisely matched the onset delay of the iontophoretic pulse used during pairing. The "ON-OFF" ratio was unchanged in the unpaired position. The modification in the paired position was still present $1 \mathrm{~h}$ after the end of the pairing procedure, at which time the neuron was lost. Calibration bars: $1 \mathrm{~s} ; 20 \mathrm{ap} \mathrm{s}^{-1}$. (D) Intracellular pairing (adapted from Frégnac et al., 1994b). Simple cell recorded intracellularly in vivo in a 10-week-old kitten. Averaged composite potential evoked by the onset of the stimulus in the $\mathrm{ON}$ subfield ( $\mathrm{C}$ : control, 21 trials). During pairing ( $\mathrm{P}$, black line) the stimulus onset was paired with a depolarizing pulse (200 ms, 1.2 nA, 30 associations). A significant potentiation of the PSP was induced after pairing (thin line: control PSP, thick lines: after pairing at 1, 3, 15, and $35 \mathrm{~min}$ ). The unpaired OFF response in the OFF subfield (not shown), the resting membrane potential ( $-67 \mathrm{mV}$, dotted lines) and the input resistance $(30 \mathrm{M} \Omega$ ) were unchanged following pairing. Calibration bar: $100 \mathrm{~ms}$. be given in those early days. Note that the later use of intrinsic imaging (Sengpiel et al., 1999; Tanaka et al., 2006) allows now to conclude that the reorganization of orientation maps was caused indeed by the expansion of domains maximally responding to exposed orientation as well as the strong reduction of responses to unexposed orientations. 
We applied our protocol of associative conditioning to demonstrate plasticity of orientation and direction selectivity during the time of recording of single cortical cells (Frégnac et al., 1988, 1992). The response of the recorded neuron was artificially reinforced during the presentation of a given orientation/direction ( $\mathrm{S}+$ ) and suppressed while presenting a different (but fixed) orientation ( $\mathrm{S}-$ ) through the same eye (see Figure 2A, left panel). Orientation tuning measurement was used to quantify the generalization of the effects to stimuli other than those used during the conditioning (column of polar plots in Figure 2A, right panel). A significant polar asymmetry favoring the $\mathrm{S}+$ preference domain was observed in $30 \%$ of conditioned cells, leading to a displacement of the peak of preferred response toward the reinforced orientation/direction.

As a general rule, these changes in tuning selectivity appeared to be linked to the competitive imbalance imposed between the two orientations presented during pairing: independently of their angular separation, a gain in responsiveness was observed around the "positively-reinforced" stimulus, whereas a loss was observed around the "negatively reinforced" one, leading sometimes to the total eradication of the initial visual response. However the amplitude of the orientation shift was related to the initial selectivity of the neuron: the probability of observing large changes in orientation preference (up to $90^{\circ}$ ) was significantly higher in initially weakly oriented neurons than in already selective ones, suggesting that most changes resulted from up- and down-regulations of pre-existing responses (Frégnac et al., 1988, 1992). This shift in functional preference could reach up to $90^{\circ}$ for orientation and corresponded to the de novo emergence of a new directional selectivity at the peak of the critical period (see example in Figure 2A). Our findings were replicated in kitten (Greuel et al., 1988) and later in adult cat cortex (McLean and Palmer, 1998), using a pharmacological control of postsynaptic activity. The phenomenology of the reported functional changes were supportive of the BCM theory predictions: (1) the largest changes were observed in cells which were the less selective and the most totipotent to stimulus features, and (2) changes were more readily observed in immature than in already specialized cortex, reflecting a dependency of the "floating plasticity threshold" on past experience.

\section{Ocular dominance plasticity}

Unilateral eye closure by lid suture performed from the third postnatal week quickly produces a dramatic change in cortical binocularity, i.e., most visual cortical neurons respond exclusively to the open eye (Wiesel and Hubel, 1963). Binocular competitive interaction between visual inputs for dominance of central connections appears to be a major mechanism involved in the effects of monocular deprivation at the cortical level: the closure of one eye produces more drastic changes than binocular closure itself. Following monocular deprivation, cortical cells become dominated or exclusively driven by the open eye, whereas preventing binocular vision by dark rearing does not affect ocular dominance (review in Frégnac and Imbert, 1984). Using moving stimuli, we simulated the effect of imbalance between the two eyes by alternately driving the same cell to a high level (S+) of activity through one eye, and a low level ( $\mathrm{S}-$ ) of firing rate through the other eye, and studying the effects on ocular dominance after 15-80 imposed associations (Shulz and Frégnac, 1992). Figure 2B illustrates a case where the spatio-temporal profile of the response to the "reinforced eye" - in addition to its magnitude - was altered: a new peak appeared as the result of an increase in responsiveness and was restricted to the previously unresponsive flank of the receptive field (delineated by filled triangles in Figure 2B) where iontophoresis had been applied concurrently with visual stimulation.

\section{Spatial organization of ON-OFF responses}

The covariance-based protocol was also adapted to control the plasticity of the spatial ON-OFF organization by inducing changes in the ON-OFF balance selective to the paired location of visual cortical receptive fields (RFs) (Debanne et al., 1998). Covariance supervision was imposed alternately in the same RF position, to boost the evoked response to a "high" level of firing (S+ pairing) for the ON (or OFF) presentation of a light bar, and, in interleaved trials, to reduce the response to the opponent OFF (or ON) feature to a "low" level (S- pairing). These differential pairings were performed iontophoretically during juxtacellular recordings (Figure 2C; Debanne et al., 1998) or intracellularly by current injection (Figure 2D; Frégnac et al., 1994b; Debanne et al., 1995). In agreement with the covariance hypothesis, they resulted in long-lasting changes of the $\mathrm{ON}$ vs OFF balance, favoring the response (ON or OFF) which had been paired with the "high" level of imposed activity.

Modifications consisted mostly of the strengthening and/or weakening of short and long-latency responses (100-800 ms); the amplitude change was on average half of that imposed during pairing. In a few cells, the de novo expression of a supra-threshold response was induced for an initially ineffective visual stimulation. Most modifications were observed in the paired position, and restricted to that region of the RF, suggesting that they probably resulted from selective changes in the transmission gain of the synapses which were activated during pairing. In a few cells, a fixed delay pairing procedure was applied, in which the iontophoretic current pulse application lagged behind the presentation or the end of the visual stimulus by a few $100 \mathrm{~ms}$, and some of the conditioned cells retained, for several tens of minutes, a temporal pattern of activity with a phase lag reproducing that imposed during pairing. An example of such an effect is shown in Figure 2C, where a long-latency response develops as a recall of the imposed delayed firing. The spatial selectivity of the effect is demonstrated by the fact that the $\mathrm{ON}-\mathrm{OFF}$ balance remains unchanged in the unpaired position (right column). Our findings of induced changes in the simple/complex profile of visual cortical RFs were also corroborated by a follow-up study using a phase conditioning protocol (McLean and Palmer, 1998), where the authors observed the induction of counter-phased modulated responses to stimuli presented at the spatial phase which initially did not evoke any response («null» phase).

Most of the changes reviewed so far were produced by extracellular pairing protocols, without access to the subthreshold synaptic events which may be modified by the Hebbian pairing procedure. In these experiments, the iontophoretic pulses used to control the excitability of the conditioned cell recruited potentially two mixed effects: (1) the first one was seen during juxtacellular recordings and corresponded to direct current effects triggering or suppressing the spike initiation (through field effect at the soma); (2) the second 
one, evoked mostly during $\mathrm{S}+$ pairings, relied on rapid changes in the extracellular potassium level. A possible side-effect may arise, due to uncontrolled potassium-dependent modification of presynaptic activity and release of neuromodulators. In order to avoid or reduce these side-effects, we made, in collaboration with Attila Baranyi, intracellular recordings (most likely intrasomatic) and used direct current injection to control the postsynaptic state of activation. This allowed a more selective pairing procedure during which the visually triggered PSP was temporally associated with a concomitant depolarizing or hyperpolarizing current pulse injection into the target cell (Figure 2D; Frégnac et al., 1994b; Debanne et al., 1995). We could then measure changes of visually evoked subthreshold synaptic potentials directly and thus interpret the observed functional changes in terms of plasticity of synaptic transmission. Similar experiments were attempted in vitro in rat and kitten visual cortical slices by Yves Frégnac in collaboration with Michael Friedlander and colleagues (Frégnac et al., 1994a), where the visual input was replaced by the electrical stimulation of the optic radiation or layer II-III axons. In the majority of conditioned cells, both in vivo and in vitro, the sign of the change (potentiation or depression) of the composite postsynaptic potential was predicted by the sign of the imposed change of the membrane potential during pairing. The effects appeared associative, since they were not observed when the current pulse was applied unrelated to visual stimulation.

The exact cellular mechanisms involved in functional changes remain difficult to unravel in vivo, since one cannot separate easily increased excitation from reduced concomitant inhibition. Blocking of inhibition in vivo leads to epileptic activity, and most pharmacological dissection methods used in vitro are no longer applicable. Data comparison suggest that enhancement in the efficacy of excitatory synaptic transmission is the most likely mechanism for the LTP observed after afferent stimulation of visual pathways both in vitro (Artola and Singer, 1987) and in vivo (Komatsu et al., 1988). Similarly, in our case, postsynaptic responses during S+ pairing were probably pushed beyond the threshold level at which NMDARdependent $\mathrm{Ca}^{2+}$ flux is sufficient to induce LTP of active synapses (see Bear, 2003 for a review).

\section{PROTOCOL 2: THETA-BURST}

Plasticity of thalamo-cortical and cortico-cortical synapses was explored following a high frequency stimulation (TBS) to sites in the LGN connected synaptically with neurons recorded intracellularly in primary visual cortex area 17 . The theta-burst conditioning consists of a short high frequency sequence of five thalamic electrical stimulation pulses (each $10 \mathrm{~ms}$ apart) repeated at $5 \mathrm{~Hz}$ (every $200 \mathrm{~ms}$ ). Intracellular recordings were obtained from $49 \mathrm{~V} 1$ cells, and in 34 cases, the full protocol (thalamic TBS and visual receptive field mapping) was carried out. Our aim was to assess at the same time the plasticity of the thalamo-cortical connections in response to an electrical (extraneous) thalamic tetanus and its functional consequences on the receptive field organization of the cortical target cells.

Single shocks of the thalamus, repeated at a low frequency $(0.2 \mathrm{~Hz})$, were applied before and after TBS to measure changes in the composite synaptic efficiency of the conditioned pathway. Postsynaptic responses to 50-100 stimulus cycles were averaged to provide a baseline control. The low rate of frequency stimulation was chosen to avoid short-term synaptic adaptation (Nelson, 1991) and the stimulation intensity was set below spiking threshold. Monosynaptic (mPSP) components in the composite synaptic responses were characterized by a short latency, very little latency jitter and the ability to follow high frequency stimulation $(100 \mathrm{~Hz})$. Polysynaptic (pPSP) components were observed with diverse latencies reflecting possibly the parallel recruitment of different synaptic pathways.

To evaluate the TBS train effect, the analog synaptic response waveforms evoked before and after the TBS application were stripped from spike contamination and averaged. The waveforms were then subtracted to visualize the temporal profile of the overall synaptic change (blue traces in Figure 3 ). The $t$-test comparison $(p<0.001)$ of peak responses and integral values (see Materials and Methods) before and after TBS showed that $44 \%$ of monosynaptic EPSPs and $56 \%$ of polysynaptic EPSPs were significantly potentiated $(n=17)$, while $39 \%$ of monosynaptic EPSPs and $25 \%$ of polysynaptic EPSPs were depressed $(n=11)$, while the remaining cases were unchanged. Note that the in vivo situation differs greatly from in vitro conditions, where it is pharmacologically possible to block inhibitory pathways (through application of GABA antagonists in the bath). Most LTP/LTD in vitro studies usually focus on the rising slope of the early monosynaptic event. In vivo, the measure of the rising slope is unreliable (since contaminated by concomitant inhibition) and the full waveform underlying spike activity has to be considered. Since the functional effects result from combined modifications of mono and polysynaptic components, we were obliged to use a combination of criteria to assess PSP changes.

Potentiation of the PSP was defined in three ways: as an increase in the peak amplitude, an increase in the integral of the response or as a reduced response latency. Conversely depression was expressed as a reduction of the PSP size and/or a lengthening of its latency. The monosynaptic and polysynaptic components appeared modified in the same proportions. In six conditioned cells, the TBS application did not trigger spikes during the high frequency bursts, resulting either in an absence of change $(n=3)$ or a depression $(n=3)$. In the other cells, where TBS imposed reliable correlation between pre-post firing (see examples in Figure 4), significant changes were observed in $90 \%$ of cases. Potentiation was more readily observed than depression (61 vs 29\%) underlying the role of the postsynaptic discharge in synaptic potentiation induction.

One should note that in vivo statistical tests (parametric and non-parametric) readily show significant changes ( $p<0.001$ here). However, these numbers have to be taken with caution since not all data obey normal distributions, and differences in variance were often seen between before and after pairing. Furthermore, numerous sources of variability are not controlled in vivo, for instance changes in the EEG reflecting the global state of the preparation, or changes in on-going intracellular activity with possible spontaneous interference of "up" and "down" states, and these may result in non-stationarities (see Discussion). Only half of cells (irrespectively of statistical significance) showed changes less than $\pm 20 \%$ in PSP integral value, which attests for a high variance in the in vivo preparation. The respective proportions of cases with potentiation and depression beyond $20 \%$ reached respectively 26 and $11 \%$. 

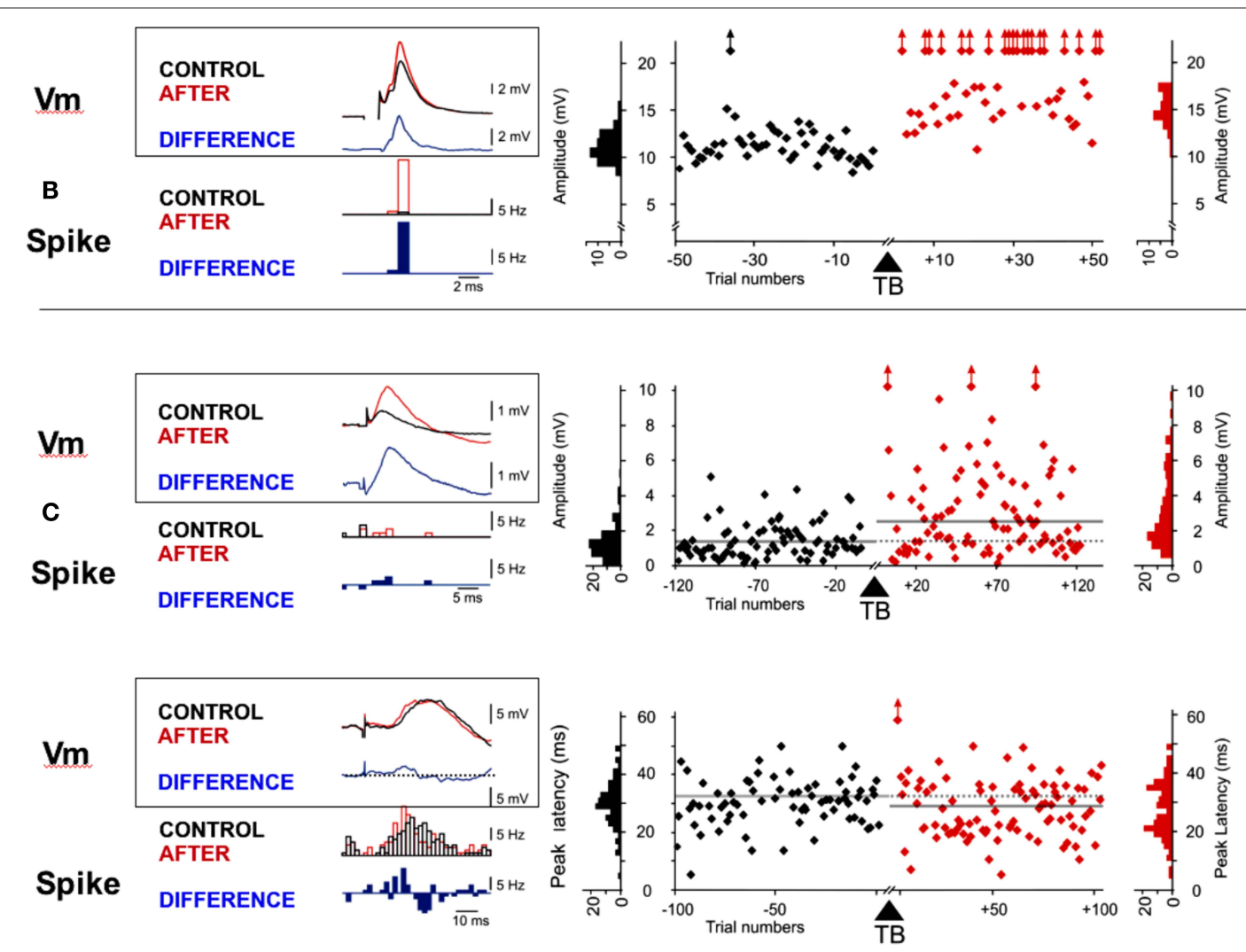

FIGURE 3 | Examples of LTP in adult visual cortex induced by thetaburst. Synaptic changes were observed on both monosynaptic (A) and polysynaptic $\mathbf{( B , C )}$ components of the test PSP by comparing the amplitude $(\mathbf{A}, \mathbf{B})$ or the latency $\mathbf{( C )}$ of the PSPs recorded before and after TBS. The left panels show overlaid averaged PSPs (at rest) and PSTHs (with a $+5 \mathrm{mV}$ depolarization) obtained before (CONTROL, in black) and after (in red) TBS The difference ("CONTROL" - "AFTER") waveforms are shown below (in blue). The right panels show the time course of the amplitudes or latencies measured on individual PSPs during the pre- and post-TBS periods. Distributions of the measured values are displayed on each side of the plots. (A) Potentiation of the peak amplitude of a monosynaptic response from $11.1 \mathrm{mV}(\sigma=1.5 \mathrm{mV})$ to $15 \mathrm{mV}(\sigma=1.9 \mathrm{mV}), p<0.001$. (B) Potentiation of a composite polysynaptic response, where the peak amplitude increased from 1.4 to $2.7 \mathrm{mV}(p<0.001)$. (C) Latency shortening $(p<0.05)$ of the peak response from 30.3 to $27.8 \mathrm{~ms}$. Note the intermittent occurrence of shorter peak latencies (18-20 ms) after TBS.
Figure 3 shows individual examples of the potentiation induced by TBS stimulation in the peak amplitude of a mPSP (Figure 3A) and in the amplitude (Figure 3B) and the latency of the peak of a pPSP (Figure 3C). For the mPSP example, the mean amplitude of the synaptic response increased by $35 \%$, from $11.1 \mathrm{mV}( \pm 1.5 \mathrm{mV})$ to $15.0 \mathrm{mV}( \pm 1.9 \mathrm{mV})$ after theta-burst stimulation of LGN input $(p<0.001)$. Figure 3B shows an example in which a pPSP was significantly increased (from 1.4 to $2.7 \mathrm{mV} ; p<0.001$ ) at the same time that the across-trial variability of responses increased, suggesting that synaptic changes could be partly due to the recruiting of new synaptic contacts that were previously ineffective. Note as a consequence the fact that the same afferent volley stimulation generally led to more spikes riding on the PSP following TBS (when tested before and after TBS at a resting state depolarized by $+5 \mathrm{mV}$ ). In two of the "potentiated" cells, the detected change was a significant reduction of the onset or peak response latency, without a change in the peak amplitude. The difference between averaged PSPs obtained before and after TBS (blue trace in Figure 3C) shows an example of potentiation mainly visible in the rising slope of the composite pPSP. As a consequence, the distribution of spikes triggered by the thalamic afferent volley (tested at a more depolarized state) was displaced toward shorter latencies following TBS (blue histogram in Figure 3C).

In order to interpret the expression of synaptic changes as a function of the interaction between pre- and postsynaptic activity, we analyzed the activity patterns imposed during the theta-burst. In many cases, the repetitive application of high frequency bursts during the TBS train resulted in the cumulative build-up of depolarization due to temporal summation of elementary responses to a single thalamic shock. This slow dynamic tendency toward cumulative depolarization was visible in the form of augmenting responses from one burst to the next, over the full train duration. These augmenting-like responses were observed during the $5 \mathrm{~Hz}$ TBS in $47 \%$ of cases, and resulted in twice as much potentiation (64.3 vs $37.5 \%$ ) and half as much depression (35.1 vs 50\% of cases), when compared to TBS trains where no cumulative recruitment was seen. This suggests that the level of depolarization reached during the high frequency thalamic burst controls the expression of synaptic potentiation, at least partly, as already shown in vitro (Sjöström et al., 2001). 


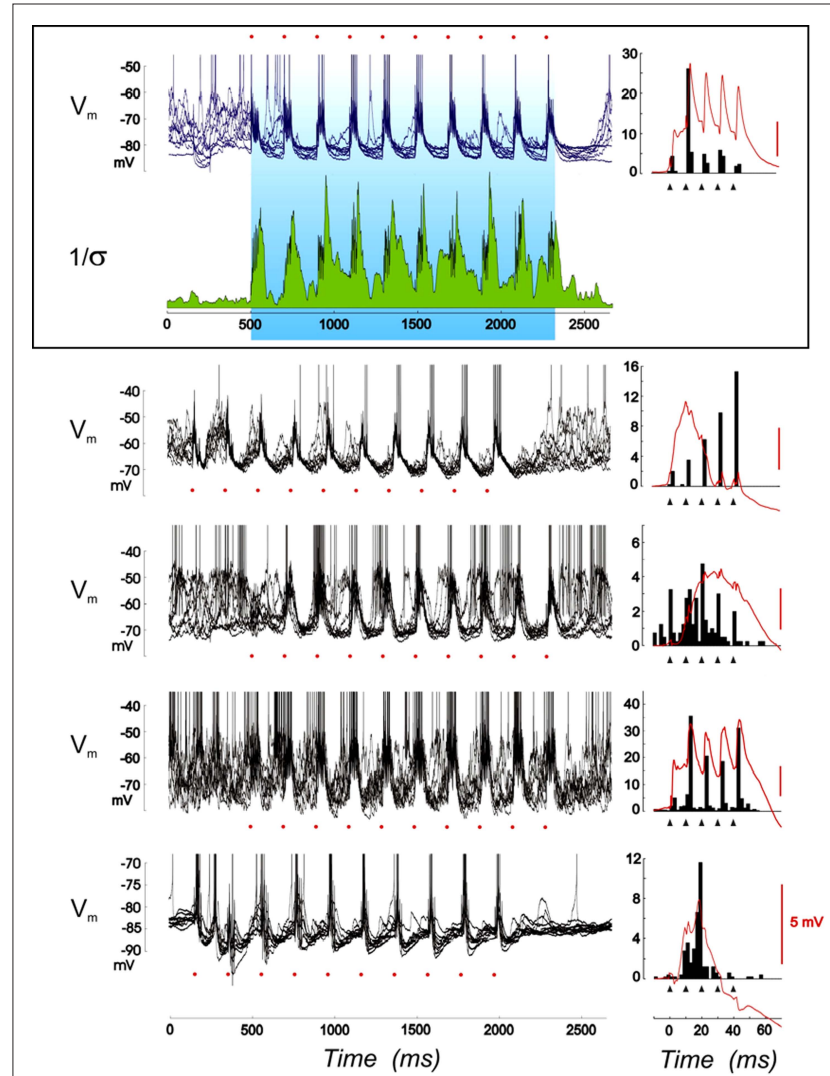

FIGURE 4 | Changes of covariance between pre- and postsynaptic activities imposed during theta-burst. For each of the five cells presented, the voltage traces are synchronized with the onset of each theta-burst train and repetitions are overlaid. The reproducibility of the recorded waveform, shown for the first cell only (inset, second row from the top), is high, especially in-between the high frequency bursts, whose individual onsets are underlined by red dots. The variability reduction in the $\mathrm{Vm}$ dynamics is quantified by the inverse of the standard deviation $(\sigma)$ of the waveforms computed over the 25 TBS repetitions (1/ $\sigma$ index, green plot) and shown with the voltage waveform for one of the cells (top inset). In the right column, the mean voltage waveform (in red) synchronized with each burst onset is shown for the duration of the burst and overlaid with the mean spike pattern imposed by the high frequency volley (black filled histogram). Black filled triangles show the occurrence of individual electrical thalamic shocks.

In contrast to these highly phasic activation periods, between bursts, a clamp of the Vm trajectory was observed resulting in the silencing of postsynaptic activity (see also Kara et al., 2002). This effect is apparent when comparing overlaid voltage traces synchronized with the TBS onset. The second row in Figure 4 illustrates a drop in variability of the stimulus-locked voltage waveform: the inverse of the standard deviation $(1 / \sigma)$ increases transiently with each burst (due to the forced positive covariance imposed by the brief tetanus) and progressively stabilizes to a high level during the time course of the repolarizing phase separating successive bursts. Consistently from cell to cell, TBS was efficient to drive the postsynaptic firing at a theta-rhythm, with tight positive correlation epochs within each high frequency burst, while suppressing responses to all inputs that may spontaneously occur between bursts. In most cells, the correlation between the thalamic electrical shock (triangles in Figure 4, right panel) and TBS-induced postsynaptic spikes was increased within the bursts, resulting in a tight control of spike timing. This observation suggests that TBS is a reliable way in vivo to favor STDP-like plasticity processes at positive presynaptic-postsynaptic delays (causal STDP). Note nevertheless that the spontaneous changes in the level of on-going activity at the time the burst is applied may affect the voltage-dependence of the synaptic responses during individual shocks of the TBS pairing, and possibly recruit additional factors controlling the expression of plasticity (Sjöström et al., 2001).

Another interesting observation from the study of postsynaptic activity during TBS is the correlation often observed in potentiated cases between the spike distribution pattern imposed during the TBS and the temporal profile of the synaptic subthreshold modification (Figure 5A). Figure 5B illustrates the time-course similarity ("isomorphism") between the forced PSTH pattern and the voltage difference curve obtained by subtracting the average PSP before and after TBS. This observation is highly reminiscent of the earlier report during covariance-based protocols that the fine temporal time course of multiple presynaptic/postsynaptic spike interactions imposed during prolonged high frequency firing (S+) sculpts a memory-like recall in the temporal waveform of the induced functional change (i.e., expressed after the pairing). For comparison, the isomorphism found in positive covariance-based protocols with static ON- or OFF-stimuli is illustrated in Figure 5C (see Figure 12 in Debanne et al., 1998).

To unambiguously demonstrate the importance of the timing of the postsynaptic action potential generation with respect to the arrival of the presynaptic afferent volley, we decided to submit 11 cells (which were also all tested with a simple TBS protocol) to a hybrid TBS-Hebbian conditioning protocol, where postysnaptic firing was imposed by an external supervisor (the experimenter), in a manner equivalent to $S+$ pairing) synchronized with the TBS train (imposing the presynaptic pattern). 25 such "TBS_S+" protocols were carried out by selecting one (and only one) of the five thalamic shocks of a given rank in the high frequency burst for a given cell, and injecting a few milliseconds later an intracellular current pulse strong enough (0.5-1.0 nA) to reliably trigger a postsynaptic spike. Delays from 2 to $8 \mathrm{~ms}$ were explored in such a way as not to interfere with the following thalamic shock. Thus the postsynaptic Hebbian supervision signal was applied at the same temporal phase for all the bursts applied during the TBS train and precisely time-locked to the presynaptic afferent volley. As shown in the three examples of the average postsynaptic spike pattern imposed during the TBS burst (green PSTHs in Figure 6), the addition of such a depolarizing current pulse (red dot) drastically reshaped the timing control of the postsynaptic firing during TBS: it changed spiking probability selectively for the first thalamic shock (cells A, B, and C of Figure 6, left), the second thalamic shock (cell B of Figure 6, middle), the third thalamic shock (cell $\mathrm{C}$ of Figure 6, middle) or the fifth thalamic shock (cells B and C of Figure 6, right), according to the chosen phase of the intracellular injection pulse.

Figure 6A shows a case in which a large potentiation was induced by pairing conditions in which the postsynaptic supervised spike followed the presynaptic spike by a few milliseconds, a result consistent with in vitro STDP. Figures 6B,C illustrate two other cases where the application of the intracellular current injection 


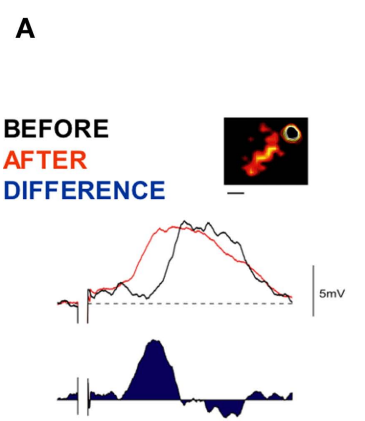

B
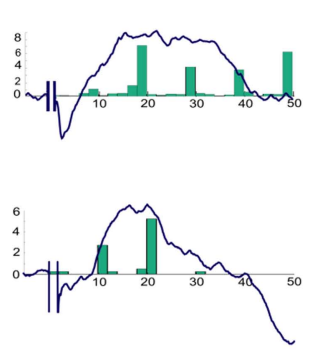

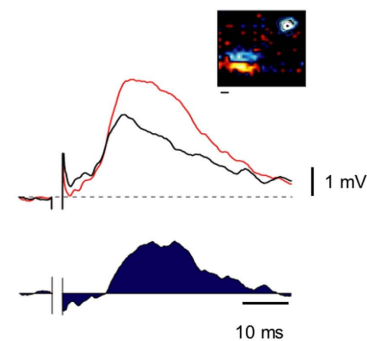

$10 \mathrm{~ms}$
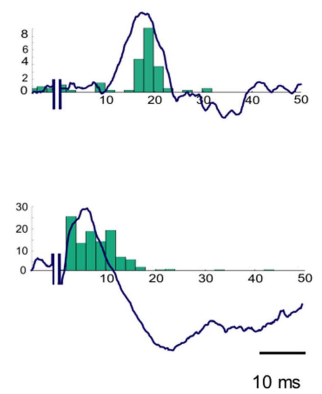

c
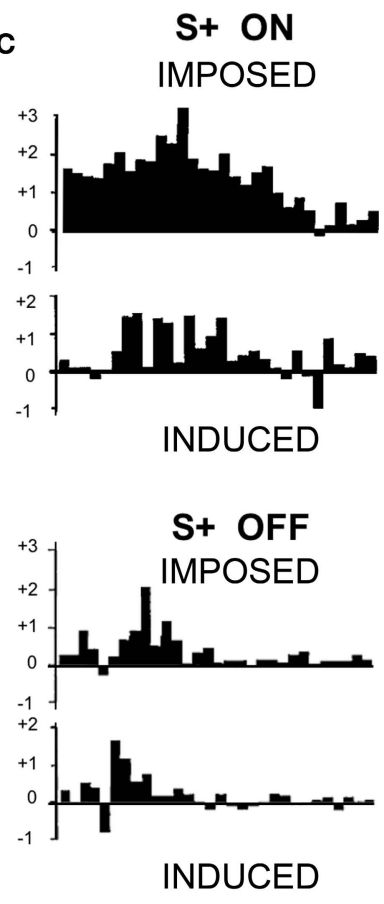

FIGURE 5 | Similarity between recalled and imposed postsynaptic patterns. (A) Examples of synaptic changes observed in the case of two cells. Insets represent the respective positions of cortical and thalamic RFs. The difference "AFTER-BEFORE" waveform (blue) shows the time course of the potentiation, corresponding to a shortening of latency (left) or an increase in peak amplitude (right). (B) Isomorphism between recalled and imposed postsynaptic patterns during TBS: The mean difference curves obtained for four different cells are superimposed with the mean spike pattern imposed during the conditioning bursts. This is done by realigning the trigger event of the control and post-TBS responses with the first thalamic shock in each high frequency burst applied during TBS. Note the similarities between the imposed spike distribution (green) and the temporal profile of the PSP change (blue). (C) Isomorphism between recalled and imposed postsynaptic patterns during $\mathrm{S}+$ covariance-based protocols (adapted from Debanne et al., 1998). Upper panel, imposed effects of S+ pairings of ON-responses. The PSTH changes ("DURING pairing" minus "BEFORE pairing"), synchronized with the stimulus onset have been normalized relative to the mean count of each bin estimated by averaging responses before and during pairing. Lower panel, induced effects. In this latter case, the PSTHs represent "AFTER" minus "BEFORE" changes. Note for each condition (top, ON responses, bottom, OFF responses) the similarity in the time course of the activity change pattern (increase for $\mathrm{S}+$ and decrease for $\mathrm{S}-$ ) locked with the stimulus onset. On average, the amplitude of the induced change is half that of the imposed change. time-locked to the fifth pulse of the afferent high frequency tetanus led to a significant potentiation, whereas similar supervised pairing time-locked to the intermediate part of the bursts resulted in depression (middle panels in Figures 6B,C). From these results, we can draw the conclusion that reshaping the postsynaptic pattern during TBS can transform depression effects into potentiation, or change the balance between excitation and inhibition, and that the multiple interactions that coexist during the high frequency burst are responsible for the observed changes. As already shown in vitro, the impact of these multiple pre-post spike interactions in controlling the sign of plasticity may depend on the rank order of the postsynaptic spikes with respect to the presynaptic multiplets (Froemke and Dan, 2002; modeled by Pfister and Gerstner, 2006).

A second aim of our experiments was to search for possible functional consequences of the plasticity induced by the TBS, in particular by looking at reconfiguration of the spatio-temporal structure of the cortical RF. We attempted to detect whether changes in location and/or extent (such as displacement, enlargement or contraction) of the subthreshold cortical RF - toward or away from the thalamic input RF - could be correlated with changes (potentiation or depression) of the synaptic response to the electrical stimulation of the thalamus. In order to do so, the cortical subthreshold RF was mapped before and after TBS by a forward correlation analysis of subthreshold responses evoked during sparse noise stimulation. For each pixel, the visually evoked PSPs were integrated over a 50-ms moving window and the reference optimal map was defined for the delay for which the RF extent was maximum. Comparison of the optimal receptive field maps recorded before and after pairings (for the same delay) was carried out on a pixel-by-pixel basis. The two largest individual cases of RF modification are presented in Figure 7. In the first case (Figure 7A), a clear increase in the MPSP amplitude was induced after TBS and this synaptic change was correlated with an enlargement of the subthreshold cortical RF. When comparing the optimal X-Y maps of the cortical subthreshold RF measured before (top left in Figure 7A) and after (bottom left) TBS, the functional change is visualized as a lateral spread of the cortical responsive zone, which invades part of the region of the visual field in which the LGN input was localized (yellow contour). The second example in Figure 7B shows modifications of the composite synaptic response to a test electrical stimulation of the thalamus (left column) and visual RF changes (right column) induced by four successive TBS_S+ pairing protocols in the same cortical cell. In this latter case, spatial stability of the RF was observed when the synaptic response was unchanged 


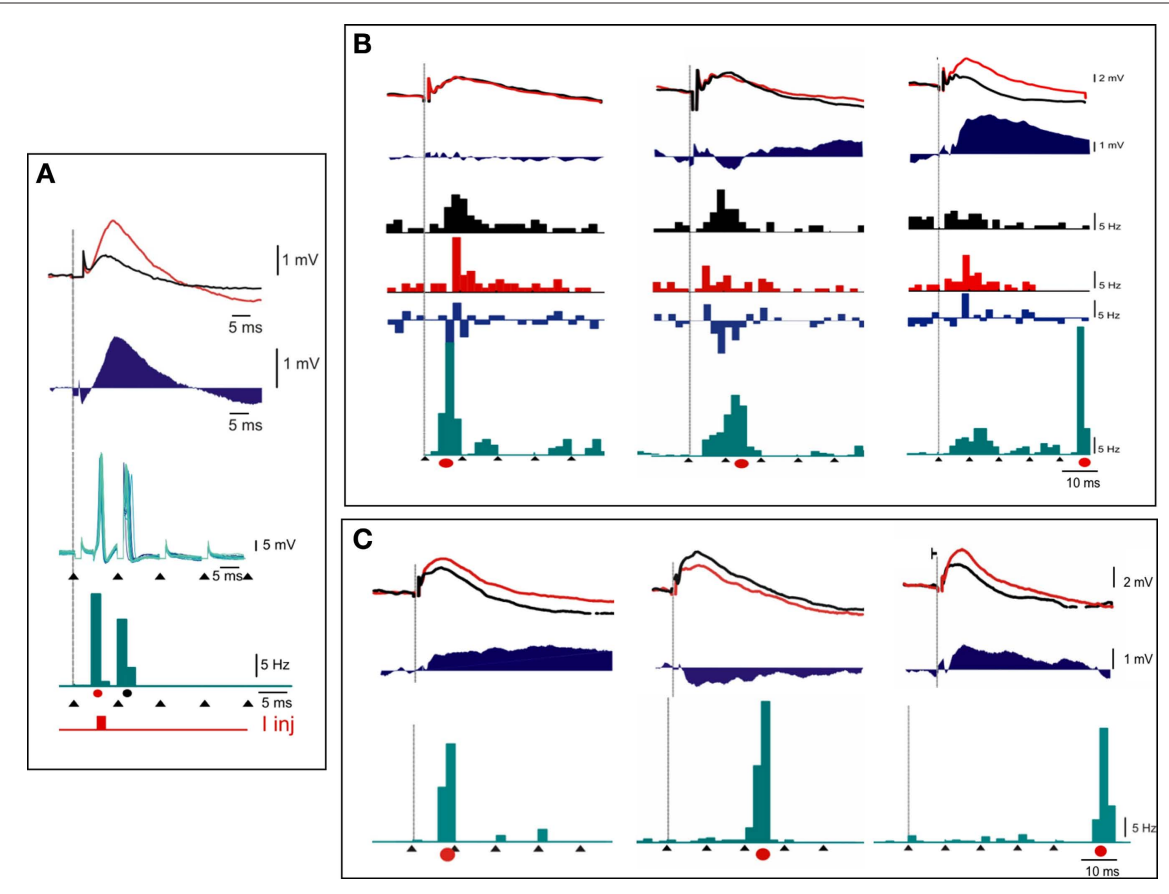

FIGURE 6 | Interaction between theta-burst and supervised Hebbian pairings: case examples. Three examples of the effects of TBS_S+ protocols, where a brief $(4 \mathrm{~ms})$ intracellular current pulse is added in phase with the 1st [cells (A), (B), and (C)], 2nd or 3rd [cells (B) and (C)], or 5th (cells (B) and (C)] electrical shock of the high frequency thalamic burst (black arrow heads). Both averaged PSPs (upper graphs) and PSTHs (lower histograms), recorded in response to a low frequency electrical thalamic shock (vertical line) before and after pairing, are represented with the same color codes [black for CONTROL, red for AFTER conditioning, and blue for difference ("AFTER"-"BEFORE")]. The imposed spike pattern during the burst is represented below, with the same temporal scale in green, and the current pulse occurrence during S+ pairing is indicated by a red dot. Note that in one of the cells (C), the hybrid conditioning results in potentiation when the intracellular current coincides with the first shock, depression when it coincides with the third shock. Potentiation is reinstated when the current pulse coincides with the fifth shock. See text for detailed comments.
A

BEFORE

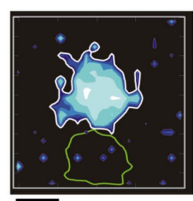

AFTER

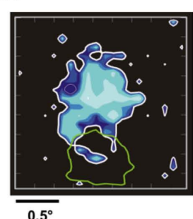

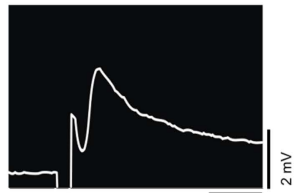

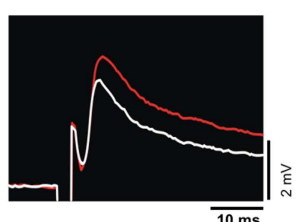

B $\Delta(\mathrm{RF})$
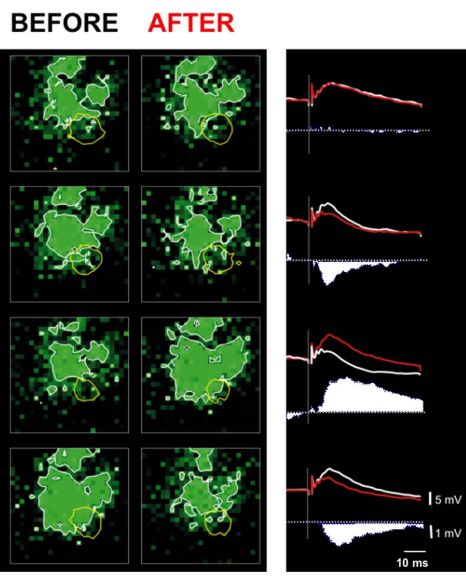

$\Delta(\mathrm{PSP})$

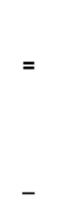

$+$

FIGURE 7 | Correlation between synaptic change induced by TBS and the spatial reorganization of the cortical RF. (A) Cell 1. The two rows show

respectively the subthreshold RF map of a V1 cell (left inset, in blue) and its mean PSP response to an individual LGN shock (right inset), observed BEFORE (top) and AFTER (bottom) thalamic TBS conditioning. In the right inset, the white and red records are respectively the PSP taken before and afterTBS. In the left inset, note in the visual X-Y maps, afterTBS, the enlargement of the subthreshold cortical RF in a spatial region in overlap with the LGN discharge field location (green contour). (B) Cell 2. Synaptic and spatial RF changes for anotherV1 cell submitted to successive conditioning protocols in which the high frequency thalamic bursts were paired with an intracellular current injection pulse (TBS_S+). Visual maps (left

column) and PSPs (right columns) are displayed in the chronological order from the top to the bottom. The 1st row depicts results obtained after aTBS_S+ (paired with the first pulse of the burst). For the second, third, and the fourth rows, the intracellular pulse was paired respectively with the second, fifth, and fourth pulse of the bursts. After each conditioning, the symbols (on each side of the figure) indicate the signs of the RF extent (left, "+" for expansion, " -" for contraction, "=" for unchanged) and the synaptic response (right side) changes. The difference "AFTER" minus "BEFORE" of the test responses are shown as filled poststimulus time waveforms. Note that the successive invasion and withdrawal of the cortical RF (green contour) from the visual LGN discharge field (yellow contour) are consistent with the sign of the synaptic changes. 
(Figure 7B, first row), whereas enlargement or contraction of the cortical subthreshold RF were correlated respectively with TBSinduced synaptic potentiation (Figure 7B, third row) or depression (Figure 7B, second and fourth rows).

The relative scarcity of these correlated observations at the single cell level (two significant cases only out of $34-6 \%$ ) is certainly constrained by the fact that thalamic and cortical RFs centers were most of the time in spatial offset and the effects too small to reach statistical significance at the single cell level (but see population study below). Another limitation of our protocols is that the duration of intracellular recording did not allow us time to test for possible occlusions (suggestive of shared input) between the electrical stimulation and visually-induced synaptic activation. We could not be sure that the contingent of synaptic responses conditioned by the TBS were part of the afferent thalamo-cortical set recruited during the mapping of visual responses. Because of the time variability of excitability and absolute levels of visual responses (that may explain minute modifications of the receptive field contour), we opted for a population analysis of the effects, presented below, rather than for a cell-by-cell analysis.

To further analyze these results, we quantified the regional changes of the spatial profiles of the optimal X-Y RF maps for each cell (Figure 8) and then applied a Fourier analysis of orientation-selective and direction-selective polar plots (the so-called "sensitivity-direction-orientation" SDO analysis in Wörgötter and Eysel, 1987; see Materials and Methods). For this analysis, the center of gravity of the RF was determined before applying TBS application and its displacement was followed thereafter. All changes were plotted in a polar coordinate system, centered around the cortical RF and whose $0^{\circ}$ axis was defined by the vector linking the cortical and thalamic RFs. When pooling all the cells of the same class (see below) together and adding the observed anisotropy vectors, the SDO analysis gave us two quantifications of a global trend: an IA and a polar measure of the Directionality $(\Theta)$ of the changes observed at the population level. These indices correspond respectively to the norm and the direction of the mean displacement vector of the center of gravity of the cortical RF.

Most remarkably, in spite of their low statistical significance at the single cell level, three coherent changes in visual receptive field organization became apparent at the population level, when the visual changes were grouped and averaged across cells which showed comparable TBS effects. Figure 8 illustrates, from left to right, the polar distribution of the visual effects pooled separately for three categories of cells, which were found to be "unchanged," "potentiated," or "depressed" by TBS. As shown by the vector sum of the individual cell changes (lower row in Figure 8), the center of gravity of the subthreshold cortical RFs of "TBS-potentiated" cells was on average displaced toward the LGN-RF location $\left(\Theta=-13^{\circ}\right.$, red vector). In contrast, the same vector sum applied to cases of "TBS-depressed" cells showed a shift away from the thalamic RF location $\left(\Theta=-229^{\circ}\right.$, blue vector in Figure 8). No significant displacement was found for cells whose test synaptic response was unchanged by TBS (black vector). We conclude that the functional $\mathrm{RF}$ reorganization that we observed most likely results from selective potentiation or depression of synaptic responses triggered by visual input in overlap with the presumed RFs of the TBS-conditioned thalamic fibers/cells.

\section{DISCUSSION}

This comparative overview of previously published covariancebased studies and our new STDP-like theta-burst pairing experiments shows that, although synaptic changes can be induced in vivo during the recording time of single cells in both cases, the former type of protocols generally leads to larger functional effects
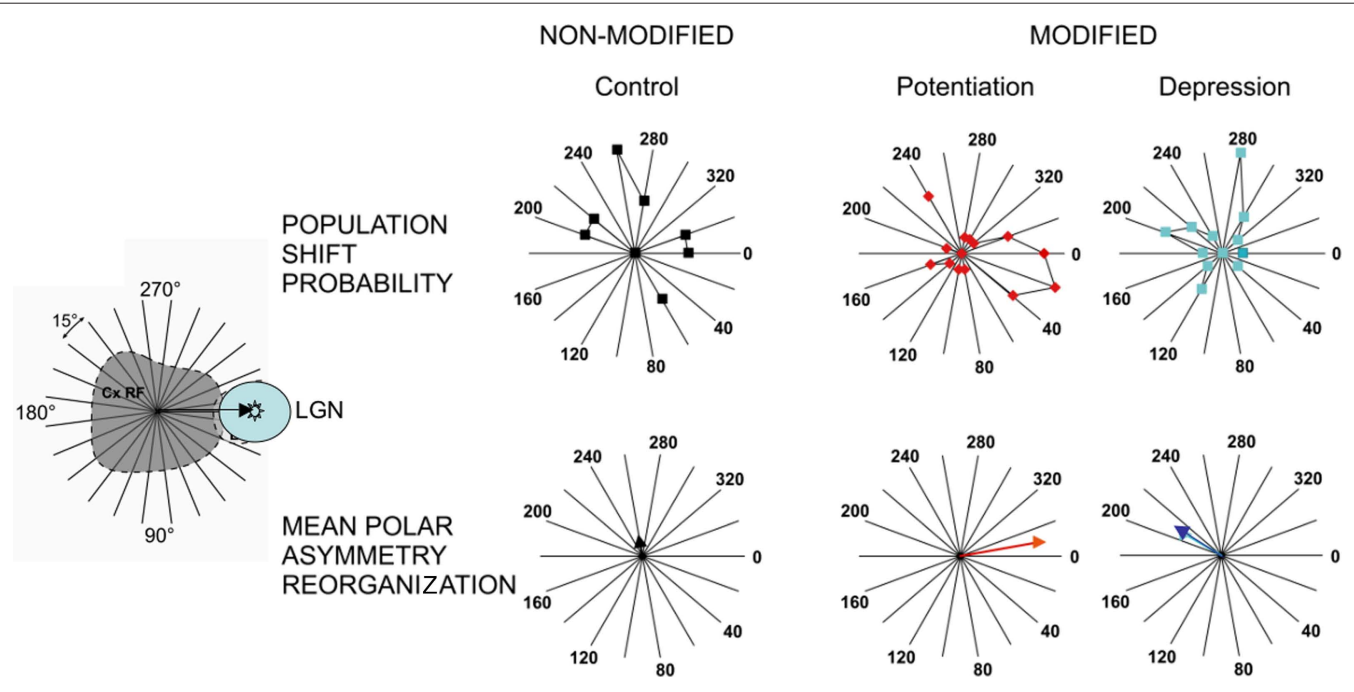

FIGURE 8 | Sensitivity-direction-orientation analysis of the spatial reorganization of RFs induced byTBS: Population study. To quantify the change of the RF shape, a polar SDO analysis was carried out on the visual RF maps obtained before and afterTBS (for details, see Materials and Methods and text). Cells have been separated according to the TBS effects on the test synaptic response to a LGN electrical shock in three groups: from left to right, not significantly modified (black), TBS-potentiated (red) orTBS-depressed (blue). Upper panel shows the mean polar plots of RF anisotropy changes, and the lower panel gives the weighted displacement vector (TBS-Non_Modified: IA = 6.6\%; DA = 81\%; TBS-Potentiated: $\mid \mathrm{A}=31.8 \%, \mathrm{DA}=-13^{\circ} ;$ TBS-Depressed: $\left.\mid A=16.3 \%, D A=229^{\circ}\right) .0^{\circ}$ indicates shift of the recorded cortical RF center toward the LGN-RF center, as shown by the arrow in the left cartoon. 
at the single cell level than those produced by STDP-based paradigms, at least in the adult cat cortex (see also review in Shulz and Jacob, 2010).

While TBS-induced functional effects that can be unambiguously correlated with the artificial-stimulus induced synaptic change are rather scarce, our experiments demonstrate that such a stimulation protocol can indeed produce significant synaptic changes in thalamo-cortical and cortico-cortical connections in adult V1. Although it is generally thought that plasticity is frozen in the anesthetized and paralyzed animal in the absence of some supervising or behavior-related signal (Hein et al., 1970, 1979; Buisseret et al., 1978; Frégnac, 1987), this finding per se may not be so surprising, since the experimenter imposes an extraneous presynaptic regime by stimulating thalamic afferents in a forced manner. Indeed, our intracellular recordings show that theta-burst trains can impose paroxysmic correlation states between thalamic and cortical activities during the time of the conditioning train, which differ strongly from those evoked by a natural sensory drive. Figure 4 shows several examples of cortical cells, monosynaptically activated from the thalamus, where the covariance between pre- and postsynaptic activities is controlled tightly during the burst itself: a succession of fast pre-post spiking events is observed during the burst (right column), while polysynaptic activity in the cortex is suppressed during the profound hyperpolarizing phase following and separating each burst at a theta frequency (see also Kara et al., 2002). During this silenced period, the cortex is clamped whereas the thalamic fibers still provide an on-going bombardment and the global activity pattern during the full conditioning train shares a strong similarity with alternate $S+/ S-$ pairings in supervised covariance-based protocols. In addition, each following tetanic burst may benefit from the waning of presynaptic depression triggered by the after-burst GABAB inhibition, whose duration is generally shorter than the theta-rhythm period (Molyneaux and Hasselmo, 2002).

Taken together, these observations indicate that the dynamic changes imposed in the covariance between pre- and postsynaptic activities may be the key factor in predicting the outcome of any conditioning protocol. This interpretation fits with our data, old and new, and can be related to other pioneer adult learning experiments in the awake behaving animal, whether supervision is imposed externally by the experimenter (Calhusac et al., 1991), or is mediated via self-generated attention related modulatory signals as shown in auditory (Ahissar et al., 1992, 1996) and somatosensory (Wang et al., 1995) cortex. In particular, Ahissar and collaborators elegantly applied cross-correlation techniques to the study of plasticity of "functional connectivity" between pairs of neurons in the auditory cortex in awake monkeys performing a sensory discrimination. The correlation of activity between two neurons was artificially controlled by immediately activating the target cell of the pair (the postsynaptic cell) by the presentation of its preferred auditory stimulus, each time the other cell fired spontaneously. Under these positive covariance conditions, reversible changes in functional coupling could be induced only when the animal was attentive to the tone used to control the activity of the postsynaptic cell. These changes were short-lived, lasting only for a few minutes, but, most remarkably, followed the covariance hypothesis predictions: potentiation of the functional link was induced when the coupling measured by cross-correlation techniques ("effective connectivity" in Aertsen et al., 1989) was increased during the pairing protocol; conversely, depression was observed when coupling was effectively reduced during the Hebbian association period.

Other interesting parallels can be made between the various protocols presented here and specific properties of STDP demonstrated in vitro, and may account for shared effects at a mechanistic level. For instance, during paired recordings in vitro the STDP efficiency in inducing LTP has been shown to require an extension of the pairing constraints not only to two precisely phase-locked events, but to higher-order patterns such as triplets (of the type "post"-"pre""post" or one "pre", two "post" according to Sjöström et al., 2001 and modeled by Pfister and Gerstner, 2006). In our S+ protocols in vivo, the covariance change is maintained for durations longer than $50 \mathrm{~ms}$, and pairings with multiple postsynaptic spikes do occur. In our hybrid theta-burst and supervised Hebbian conditioning, potentiation effects are more easily revealed when the spiking is imposed for the last (fifth) stimulation shock during the burst. If one compares this latter situation to the reinforcement of the first thalamic shock in the burst, the pairing pattern is closer to a "postpre-post" than to the "pre-post-pre" configuration known to be less effective in inducing potentiation (Froemke and Dan, 2002; Pfister and Gerstner, 2006). The forced S+ pairing at the end of the burst also benefits from the cumulative depolarization which builds-up in some cells during the high frequency tetanus (see examples 1, 3, 4 from the top in Figure 4). It has been shown that only bursts of action potentials above a critical frequency $(100 \mathrm{~Hz})$ induce dendritic spikes (Larkum et al., 1999). Action potential bursts need also to exceed roughly the same critical frequency to induce STDP (Kampa et al., 2006), suggesting a threshold requirement for dendritic spikes, and it is likely that the theta-burst pattern in our protocol attained that frequency. Consequently, the enhanced efficacy of the pairing during the last shock of the burst could be explained by the fact that regenerative $\mathrm{Ca}^{2+}$ dependent dendritic potentials are particularly evident in some cells by the end of a burst (see Figure 1 in Larkum et al., 1999). For the negative covariance change, one should also note the similarity between our S- protocols and some variants of STDP protocols used by Sjöström in vitro, where depression is observed when a hyperpolarization current is added in-between current-induced spikes during high frequency pairings. The difference still remains that during most STDP and theta-burst protocols, presynaptic and postsynaptic activities are phase-locked for each occurrence of the presynaptic spike, whereas, during covariance-based protocols, presynaptic and postsynaptic activities are controlled in two independent ways (the stimulus feature for presynaptic activity, the current-induced for postsynaptic firing).

Apart from these similarities, the functional impact of the two types of protocols seems to differ, not so much in the sign of the induced changes, but in their respective amplitude and probability of induction. Several explanations are possible for why the ratebased covariance manipulation experiments showed a much larger potential for plasticity in vivo than currently reported by thetaburst, Spike Timing-Dependent and Stimulus-Timing-Dependent protocols (Yao and Dan, 2001; Fu et al., 2002). One possible reason is linked to the local supervised vs global unsupervised nature of some of the conditioning protocols. The imposition of a local 
perturbation, as engineered by the sole control of the postsynaptic firing of the paired cell in our covariance-based protocols, will induce a regional reorganization in a weakly coupled network. In contrast, a global clamp of cortical input, as imposed by the sequential presentation of distinct features in stimulus-timing-dependent protocols, may have a limited effect at the single cell level: in this latter situation, a large part of the cortical network is conjointly activated by the two sequential stimuli which often cover a large part of the visual field and the recruitment of multiple interactions may cancel the lateral spread of competitive effects. In our $\mathrm{S}+/ \mathrm{S}-$ protocols, the constraints from the rest of the network in stabilizing the columnar preference, hence the conditioned cell's preference, may be weaker, allowing the cell's response to escape locally from the global assembly behavior.

Another possible explanation is that some associativity threshold has to be reached in adult cortex for expressing plasticity, which goes well beyond the pairing of two single events. Although Hebbianlike changes have been observed in the adult auditory cortex when the awake behaving monkey is attentive (Ahissar et al., 1992), these changes are short-lived and do not compare in strength with those we observed in kitten V1 cortex. A similar reasoning may apply to STDP: since the most compelling evidence for a functional role of STDP in vivo has been mostly demonstrated during development and in Xenopus (Tao et al., 2001; Mu and Poo, 2006), one may question to what extent plasticity can be reliably revealed by using similar STDP-based protocols in vivo in adult mammalian cortex. The only comparable experiment in cat and rat V1 cortex comes from the studies from the group of Yang Dan. In a first series of experiments, Dan's team devised very ingenious visuo-visual pairing protocols where the timing of the presynaptic volley and postsynaptic volleys were indirectly controlled by manipulating the asynchronous timing of presentation of various features of the visual input (orientation, position) (Yao and Dan, 2001; Fu et al., 2002). These differential protocols relied however on the assumption that inputs representing each of the two selected stimulus features (presented sequentially) were separable in terms of presynaptic neuronal population activation, omitting to take into account the reverberant activity produced by each of the stimuli at the postsynaptic level. Furthermore, the interaction of components in the target response was not measured in these studies. A second problematic issue is that most of the functional changes that the group of Yang Dan reported at the single cell level were of moderate amplitude (mean reported value of $2.8^{\circ}$ ), well below the experimental precision of their RF or tuning curve measures $\left(12-15^{\circ}\right.$ step in Yao and Dan's study). In spite of these limitations, the visuo-visual pairing protocols were assessed at the population level, under the additional assumption that the same plasticity rule would apply across cells and high performance bootstrap techniques were used to show a significant effect at the population level. The interpretation we derive from these extracellular stimulusdependent-plasticity studies is that, in contrast to covariance control protocols, visuo-visual pairings produce almost undetectable changes at the single cell level but significant incremental changes, observable only at the population level.

More direct and sophisticated attempts have been made in vivo with intracellular techniques. In rat V1, Meliza and Dan (2006) achieved a "tour de force" experiment, in which the spatio-temporal
RF of each neuron was measured under voltage clamp conditions, before and after STDP protocols. To induce modification of the RF, the authors switched the recording to current-clamp configuration, and repetitively paired the visual stimulus at one of the four RF locations ( spaced by $11-13^{\circ}$ ) with a brief intracellular current injection (6-8 ms) that forced postsynaptic spiking. This protocol can be seen as analogous to the STDP induction protocol in rat visual cortical slices (Sjöström et al., 2001; Froemke and Dan, 2002), except that presynaptic activation was caused by visual, rather than electrical, stimulation. Modifications of the visual responses, potentiation and depression were observed within a selectivity window of $\sim \pm 50 \mathrm{~ms}$, similar to that observed in rat visual cortical slices.

Interpretation of the results obtained for positive delays is less straightforward than for negative delays and can be questioned in two ways: the first issue concerns the spatial selectivity of the effect. Meliza and Dan reported significant response modifications at the unpaired locations, which, when restricted to the potentiation cases in the paired location, were uncorrelated in sign (both potentiation and depression could be seen in the unpaired location, see Figure 4E in Meliza and Dan, 2006 and red inset in Figure 9, this paper) and often larger in amplitude than those induced in the paired location. The diversity in hetero-positional effects was found to be linked on whether the pairing was applied at the center or the flank of the RF. From a functional perspective, the location dependence of the RF plasticity they described seemed to favor potentiation of the weak parts of the RF and to facilitate shifts of the RF center to neighboring locations when stimuli at these locations were followed by postsynaptic spiking. Nevertheless, the fact that the amplitude of the hetero-positional effects was often larger than that observed in the paired position and the unusually large width of the stimulus (hence, of the spatial separation bin size between explored positions), raise the issue of the specificity of the spatial selectivity in the reported effects.

Comparison with the STDP literature is further complicated by the fact that, in Meliza and Dan's experiment, the "pre"- "post" pairing interval is defined as the interval between the peak of the visually evoked inward current and the peak of the postsynaptic action potential. This convention differs from the classical definition where the "pre"-"post" interval is defined between the first presynaptic spike or the subthreshold PSP onset, and the somatic postsynaptic spike (see for instance Figures 2D,H in $\mathrm{Mu}$ and Poo, 2006). In Figure 10 we have qualitatively realigned the results of Meliza and Dan, shown in Figure 2E of their original paper, with the classical STDP convention (i.e., removing the average delay from response onset to peak response), and plotted the STDP curve as a function of the "pre"-"post" delay (where the zero time, indicated by the purple dotted $y$-axis, signals the firing time of the first presynaptic spike in the sensory input volley). This realignment has two consequences: (1) the STDP curve obtained by Meliza and Dan would still predict significant depression for negative delays; (2) However, the outcome of the pairing for positive delays would be highly variable on a cell-by-cell basis: for a delay window of 0 to $+20 \mathrm{~ms}$, as many cases of depression $(n=4)$ and potentiation $(n=3)$ would be observed. To the difference of Meliza and Dan, we conclude that positive "pre"-"post" delays do not result in systematic potentiation in adult sensory cortex in vivo. A similar conclusion was reached in adult rat somatosensory cortex by Shulz and 

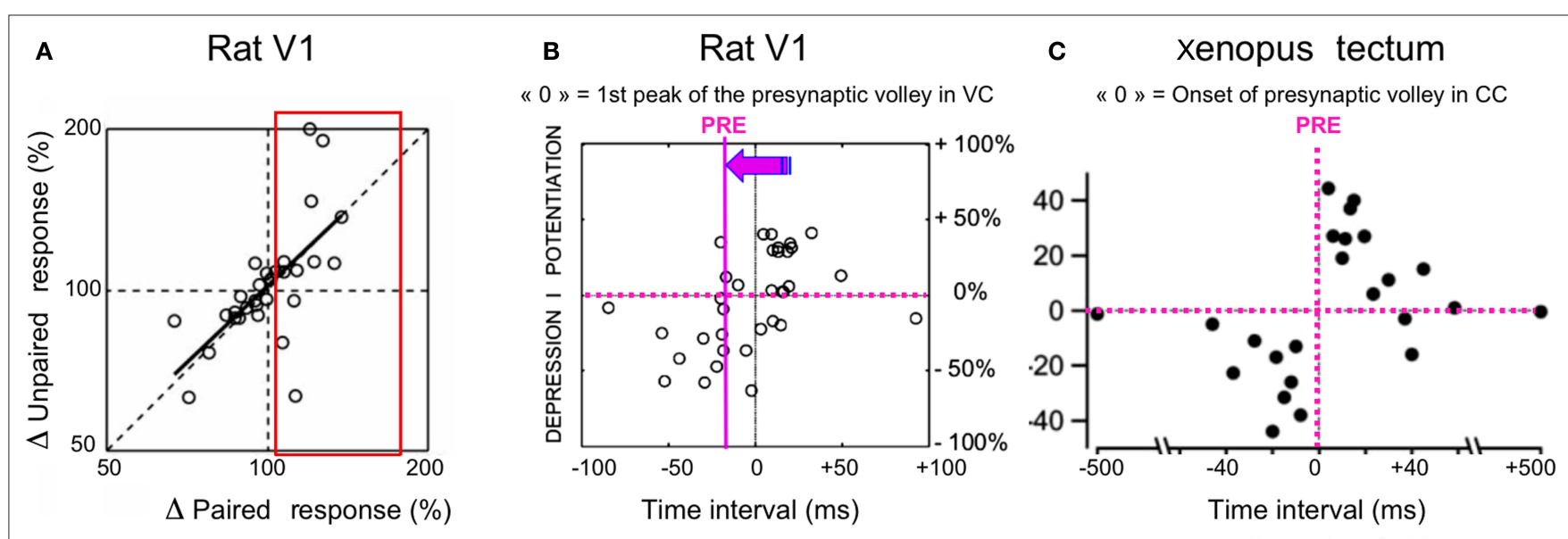

FIGURE 9 | Re-examination of in vivo STDP. (A) Rat visual cortex, taken from Figure 4E in Meliza and Dan (2006): change in unpaired RF region vs change in the paired region (log-log scale) following STDP intracellular pairings (see text for details). Each point represents an individual cell. The oblique regression line is a linear fit calculated on all cases (potentiation and depression). The red inset underlines the lack of spatial selectivity for potentiation effects, whose amplitudes are often larger for the unpaired than for the paired positions. (B) Rat visual cortex, adapted from Figure 2E in Meliza and Dan (2006): Change in amplitude of visual response at paired location as a function of pairing interval. Each symbol represents one cell. The $x$-axis coordinates of the original plot have been inverted in sign, to make the STDP curve comparable to that shown in (C). "0" represents the occurrence of the peak inward current measured in voltage clamp (VC). The purple $y$-axis, shifted to the left, represents the most likely arrival time of the first presynaptic spike. The position difference between the two-y axes corresponds to the mean delay separating the onset from the peak input. (C) Xenopus tectum, taken from Figure $2 \mathrm{H}$ in $\mathrm{Mu}$ and $\mathrm{PoO}$ (2006): STDP curve induced by repetitive single-bar stimulation in the developing retino-tectal system of Xenopus. The graph is aligned on the presynaptic volley onset and shows a clear partition of spike timing-dependent depression (negative delays) and potentiation (positive delays) of tectal responses, recorded in current clamp (CC). Each point represents the result from one experiment. collaborators together with the group of Dan Feldman (Jacob et al., 2007). In summary, these different studies show for the least that STDP-induced potentiation remains difficult to establish in vivo in adult cortex, and that associative plasticity remains dominated by selective depression.

This raises the question whether the impact of STDP for inducing incremental strengthening in functional connectivity in adult cortex has been largely over-stated in the literature. This effect, expected from the consensus achieved on the basis of the in vitro literature, may in fact be more elusive in vivo, especially in the adult cortex (review in Shulz and Jacob, 2010). This difficulty in replication may come from the usual difficulties associated with in vivo experiments, which constrain all experimental intracellular studies including ours. A fair statement should be to consider that very few unambiguous single cell cases of LTP have been published so far in adult rodent and cat sensory cortices: in general, the duration of the control periods are too short to exclude non-stationarities and slow trends in synaptic efficacies are visible in the longer postconditioning periods of some reports. Among uncontrolled factors in the anesthetized preparation, is the role of inhibition: it is seen as a suppressive gate by many, which may interfere with the dendritic spread of back propagating action potentials (Engelmann et al., 2008). Although inhibitory interneurons modulate many neuronal processes, the evidence for plasticity at inhibitory synapses remains scarce. Some studies report strengthening of inhibitory synapses in negative rate covariance regimes (Komatsu and Iwakiri, 1993; Komatsu, 1994, 1996; Holmgren and Zilberter, 2007). Spike timingdependent plasticity of inhibitory synapses has been also reported (Haas et al., 2006) as well as spike timing-dependent depression of excitatory synapses on fast spiking inhibitory interneurons
(Lu et al., 2007). An additional factor that has to be taken into account is the complex interaction between back propagating action potentials and the local excitable properties of the dendritic tree. Dendrites contain voltage-activated channels, but also they can support fast action potential-like events mediated by voltageactivated $\mathrm{Na}^{+}$channels, or slower, regenerative events mediated by voltage-activated $\mathrm{Ca}^{2+}$ channels (Stuart et al., 1997). Due to intense background synaptic activity in vivo, these conductances may have a particular impact on the spread of back propagating action potentials (Sjöström and Hausser, 2006) and eventually help or interfere with STDP induction (van den Burg et al., 2007).

The last conclusion concerns the validity of inferences that one may be tempted to make for the in vivo case from consensual plasticity rules only substantiated in in vitro conditions in developing networks. Many experimental reports point to the possibility that homeostasis rules may be exacerbated in the slice or organotypic culture preparations (review in Frégnac, 1999; Turrigiano and Nelson, 2000). The possibility for such deafferented networks of undergoing lesion-induced or posttraumatic forms of plasticity to recover an operational range of synaptic efficiency may not be so readily expressed in the intact brain. Nonetheless, deafferentation of cortical regions by peripheral lesions lead to receptive fields reorganization that are more readily explained by STDP than correlation rate-based plasticity rules (Young et al., 2007). The second main difference between the in vitro and the in vivo cases is the presence in vivo of irregular on-going activity. This bombardment ensures a basal level of correlation between spontaneous inputs (linked with the high level of on-going activity in thalamus and the profusion of recurrent intracortical circuits) and cortical cells. During sensory 

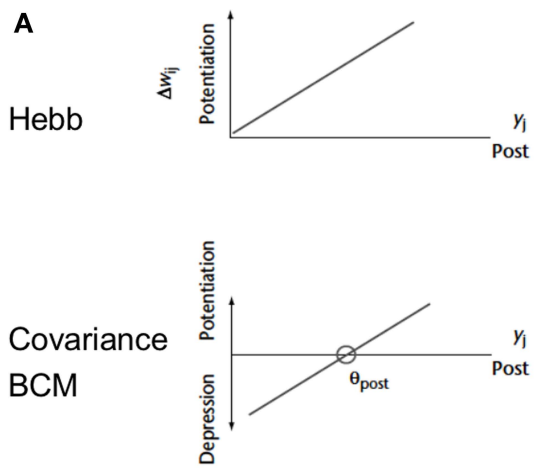

ABS

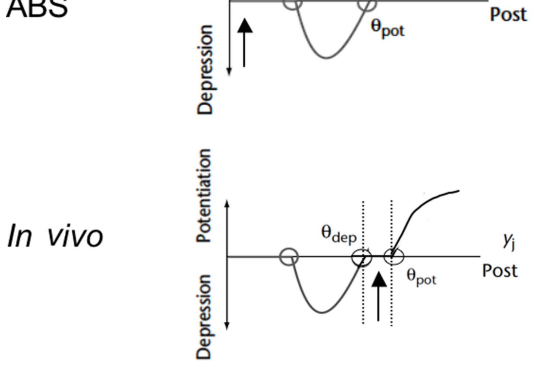

FIGURE 10 | For a diversity of plasticity rules in vitro and in vivo. (A) Generalized Hebbian forms of plasticity. From top to bottom, Hebb's rule (top) and the most often observed rules of homosynaptic plasticity established in vitro and in vivo (bottom). Each graph expresses the theoretical relationship between the induced synaptic change (positive ordinates for potentiation, negative for depression) and postsynaptic activity at the time of the association. The slope is proportional to presynaptic activity. The simple Hebbian rule predicts potentiation only. The covariance rule (second from the top) and A-B-S rule (third from the top) predict both depression and potentiation with, respectively, one

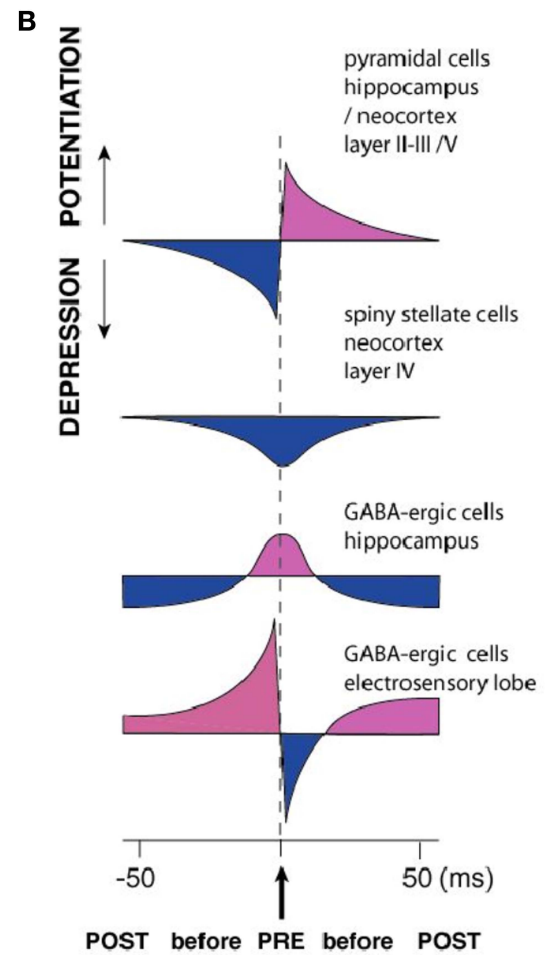

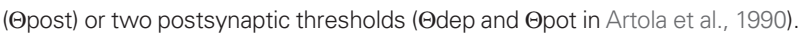
Bottom: covariance rule observed in vivo, where a dead-zone lies near the operating regime (vertical arrow) of the synapse during sensory processing (Frégnac et al., 1988; Frégnac, 1991). (B) Diversity of forms of STDP established in vitro. The induced synaptic change is expressed as a function of the temporal delay between presynaptic firing (zero time reference) and postsynaptic spike occurrence (adapted from Abbott and Nelson, 2000; Frégnac, 2002). A mirror form of STDP is also observed in inhibitory neurons of the electrosensory lobe of the electric fish (Bell et al., 1997). activation, positive co-variation between pre- and postsynaptic activities is observed without changing receptive field properties, at least in the anesthetized and paralyzed preparation (review in Frégnac and Imbert, 1984). In view of the relative difficulty of inducing acute functional changes in the intact neocortex, we proposed some 20 years ago the existence of a threshold in the expression of STDP in vivo outside the critical period, which accounts for the stability of synaptic weights during normal sensory processing (Frégnac et al., 1988, 1994a; Frégnac, 1991). The observation that synaptic or functional changes can be induced by a supervised control of presynaptic (theta-burst) and postsynaptic (STDP and Hebbian) activities, as shown here, agrees with the view that, in the intact brain, expression of plasticity would require some drastic reconfiguration of covariance or correlation changes between pre-and postsynaptic activities, which goes well beyond evoked changes by natural stimuli. Additional boosting control signals are needed, such as those self-generated by the brain under the form of attention-gated or reward-driven processes during behavioral learning, that push the correlational state detected by the synapse beyond the level reached during normal sensory processing. This "correlation change threshold" hypothesis would thus require that the covariance change between pre- and postysnaptic activities during the associative learning is large enough and is maintained long enough to push away the operational working regime of the synapse from its non-adaptive "read-only" state (bottom graph in Figure 10A, taken from Frégnac, 1991; see also Ahissar et al., 1992 for an implementation). It would be only by trespassing the required correlation change threshold(s), that the expression of association-induced functional changes would be validated in adult cortex by reward or behavior.

\section{ACKNOWLEDGMENTS}

We thank Drs Kirsty Grant and Andrew Davison for their help in reviewing this manuscript. This work has been supported by the CNRS, the DGA, the Agence Nationale de la Recherche (ANR) and EC contracts Sensemaker (FP5-2001-IST 34712), Facets (FP6-2004IST-FETPI 15879) and Brain-i-nets (FP7-2009-ICT-FET 243914). All research procedures concerning the experimental animals and their care adhered to the American Physiological Society's Guiding Principles in the Care and Use of Animals, to the French (JO 87-848), the European Council Directive 86/609/EEC and to European Treaties Series 123 and was also approved by the regional ethics committee "Ile-de-France Sud" (Certificate 05-003). 


\section{REFERENCES}

Abbott, L. F., and Nelson, S. B. (2000). Synaptic plasticity: taming the beast. Nat. Neurosci. 3, 1178-1183.

Aertsen, A., Gerstein, G. L., Habib, M. K., and Palm, G. (1989). Dynamics of neuronal firing correlation: modulation of "effective connectivity". J. Neurophysiol. 61, 900-917.

Ahissar, E., Haidarliu, S., and Shulz, D. (1996). Possible involvement of neuromodulatory systems in cortical Hebbian-like plasticity. J. Physiol. (Paris) 90, 353-360.

Ahissar, E., Vaadia, E., Ahissar, M., Bergman, H., Arieli, A., and Abeles, M. (1992). Dependence of cortical plasticity on correlated activity of single neurons and on behavioral context. Science 257, 1412-1415.

Albus, K. (1975). A quantitative study of the projection area of the central and the paracentral visual field in area 17 of the cat: I. The precision of the topography. Exp. Brain Res. 24, 159-179.

Andrew, R. D., and Fagan, M. (1990). A technique for controlling the membrane potential of neurons during unit recording. J. Neurosci. Methods 33, 55-60.

Artola, A., Bröcher, S., and Singer, W. (1990). Different voltage-dependent thresholds for inducing long-term depression and long-term potentiation in slices of rat visual cortex. Nature 347, 69-72.

Artola, A., and Singer, W. (1987). Longterm potentiation and NMDA receptors in rat visual cortex. Nature 330, 649-652.

Baranyi,A., and Feher, O. (1981). Synaptic facilitation requires paired activation of convergent pathways in the neocortex. Nature 290, 413-415.

Bear, M. F. (2003). Bidirectional synaptic plasticity: from theory to reality. Philos. Trans. R. Soc. Lond. B 358, 649-655

Bear, M. F., Cooper, L. N., and Ebner, F. (1987). A physiological basis for a theory of synapse modification. Science 237, 42-48.

Bear M. F., Kleinschmidt, A., Gu, Q., and Singer, W. (1990). Disruption of experience-dependent synaptic modifications in striate cortex by infusion of an NMDA receptor antagonist. J. Neurosci. 10, 909-925.

Bear, M. F., and Malenka, R. (1994). Synaptic plasticty: LTP and LTD. Curr. Opin. Neurobiol. 4, 389-399.

Bear, M. F., Press, W. A., and Connors, B. W. (1992). Long-term potentiation in slices of kitten visual cortex and the effects of NMDA receptor blockade. J. Neurophysiol. 67, 841-851.

Bear, M. F., and Singer, W. (1986). Modulation of visual cortical plasticity by acetylcholine and noradrenaline. Nature 320, 172-176.
Bell, C., Han, V., Sugawara, Y., and Grant, K. (1997). Synaptic plasticity in a cerebellum-like structure depends on temporal order. Nat. Neurosci. 387, 278-281.

Bi, G. Q., and Poo, M. (2001). Synaptic modification by correlated activity: Hebb's postulate revisited. Annu. Rev. Neurosci. 24, 139-166.

Bienenstock, E., Cooper, L. N., and Munro, P. (1982). Theory for the development of neuron selectivity: orientation specificity and binocular interaction in visual cortex. J. Neurosci. 2, 32-48.

Blais, B. S., Shouval, H. Z., and Cooper, L. N. (1999). The role of presynaptic activity on the ocular dominance shift in monocular deprivation: comparison of homosynaptic and heterosynaptic mechanisms. Proc. Natl. Acad. Sci. U.S.A. 96, 1083-1087.

Blakemore, C., and Cooper, G. F. (1970). Development of the brain depends on the visual environment. Nature 228, 419-478.

Brown, T. H., Ganong, A. H., Kairiss, E. W., and Keenan, C. L. (1990). Hebbian synapses: biophysical mechanisms and algorithms. Annu. Rev. Neurosci. 13, 475-511.

Brown, R. E., and Milner, P.M.(2003). The legacy of Donald O. Hebb: more than the Hebb synapse. Nat. Rev. Neurosci. 4, 1013-1019.

Buisseret, P., Gary-Bobo, E., and Imbert, M. (1978). Ocular motility and recovery of orientational properties of visual cortical neurones in dark-reared kittens. Nature 272, 816-817.

Calhusac, P. M. B., Rolls, E. T., and Marriott, F. H.C. (1991). Potentiation of neuronal responses to natural visual input paired with postsynaptic activation in the hippocampus of the awake monkey. Neurosci. Lett. 124, 39-43.

Crair, M. C., and Malenka, R. C. (1995). A critical period for long-term potentiation at thalamocortical synapses. Nature 375, 325-328.

Crepel, F. (1998). Nitric oxide and longterm depression in the cerebellum. Trends Neurosci. 21, 63-64.

Crow, T. (1968). Cortical synapses and reinforcement: a hypothesis. Nature 219, 736-737.

Debanne, D., Gahwiller, B. H., and Thompson, S.M.(1997). Bidirectional associative plasticity of unitary CA3-CA1 EPSPs in the rat hippocampus in vitro. J. Neurophysiol. 77, 2851-2855.

Debanne, D., Shulz, D. E., and Frégnac Y. (1995). Temporal constraints in associative synaptic plasticity in hippocampus and neocortex. Canad. J. Physiol. Pharmacol. 73, 1295-1311.

Debanne, D., Shulz D. E., and Frégnac Y. (1998).Activity dependent regulation of on- and off-responses in cat visual cortical receptive fields. J. Physiol. 508, 523-548.

Dudek, S. M., and Bear, M. F. (1992) Homosynaptic long-term depression in area CAl of hippocampus and effects of $N$-methyl-D-aspartate receptor blockade. Proc. Natl. Acad. Sci. U.S.A. 89, 4363-4367.

Dudek, S. M., and Bear, M. F. (1993). Bidirectional long-term modification of synaptic effectiveness in the adult and immature hippocampus. $J$. Neurosci. 13, 2910-2918.

Dudek, S. M., and Friedlander, M. J. (1996). Developmental downregulation of LTD in cortical layer IV and its independence of modulation by inhibition. Neuron 16 , 1097-1106.

Engelmann, J., van den Burg, E., Bacelo, J., de Ruijters, M., Kuwana, S., Sugawara, Y., and Grant, K. (2008). Dendritic backpropagation and synaptic plasticity in the mormyrid electrosensory lobe. J. Physiol. (Paris) 102, 233-245.

Feldman, D. E. (2000). Timing-based LTP and LTD at vertical inputs to layer II/ III pyramidal cells in rat barrel cortex. Neuron 27, 45-56.

Foeller, E., and Feldman, D. E. (2004) Synaptic basis for developmental plasticity in somatosensory cortex. Curr. Opin. Neurobiol. 14, 89-95.

Frégnac, Y. (1987). "Cellular mechanisms of epigenesis in cat visual cortex," in Imprinting and Cortical Plasticity, eds J. P. Rauschecker and P. Marler (New York: John Wiley and Sons), 221-266.

Frégnac, Y. (1991). “Computational approaches to network processing and plasticity," in Long-Term Potentiation, ed. M. B. J. L. Davis (Cambridge, MA/ London: MIT Press), 425-435.

Frégnac, Y. (1999). The tale of two spikes Nat. Neurosci. 2, 299-301.

Frégnac, Y. (2002). "Hebbian synaptic plasticity," in Handbook of Brain Theory and Neural Networks, ed. M.A Arbib (Cambridge: The MIT Press), 515-522.

Frégnac, Y., Burke, J., Smith, D., and Friedlander, M. J. (1994a). Temporal covariance of pre and postsynaptic activity regulates functional connectivity in the visual cortex. J. Neurophysiol. 71, 1403-1421.

Frégnac, Y., Debanne, D., Shulz, D. E., and Baranyi,A. (1994b). "Does post synaptic membrane potential regulate functional plasticity in kitten visual cortex?" in Long-Term Potentiation, eds. M. Baudry and J. L. Davis (Cambridge: MIT Press), 227-264.

Frégnac, Y., and Imbert, M. (1984). Development of neuronal selectivity in the primary visual cortex of the cat. Physiol. Rev. 64, 325-434.
Frégnac, Y., and Shulz, D. E. (1994). "Models of synaptic plasticity and cellular analogs of learning in the developing and adult vertebrate visual cortex," in Advances in Neural and Behavioral Development, eds V. Casagrande and P. Shinkman (Norwood, NJ: Neural Ablex Publ.), 149-235.

Frégnac, Y., and Shulz, D. E. (1999). Activity-dependent regulation of receptive field properties of cat area 17 by supervised Hebbian learning. $J$. Neurobiol. 41, 69-82.

Frégnac, Y., Shulz, D. E., Thorpe, S., and Bienenstock, E. (1988). A cellular analogue of visual cortical plasticity. Nature 333, 367-370.

Frégnac, Y., Shulz, D. E., Thorpe, S., and Bienenstock, E. (1992). Cellular analogs of visual cortical epigenesis: I. Plasticity of orientation selectivity. $J$. Neurosci. 12, 1280-1300.

Frey, U., and Morris, R. G. (1997). Synaptic tagging and long-term potentiation. Nature 385, 533-536.

Froemke, R., and Dan, Y. (2002). Spiketiming-dependent synaptic modification induced by natural spike trains. Nature 416, 433-438.

Froemke, R., Poo, M., and Dan, Y. (2005a). Spike-timing-dependent synaptic plasticity depends on dendritic location. Nature 434, 221-225.

Froemke, R., Tsay, I., Raad, M., Long, J. and Dan, Y. (2005b). Contribution of individual spikes in burst-induced long-term synaptic modification. $J$. Neurophysiol. 95, 1620-1629.

Fu, Y. X., Djupsund, K., Gao, H., Hayden, B., Shen, K., and Dan, Y. (2002). Temporal specificity in the cortical plasticity of visual space representation. Science 296, 1999-2003.

Gerstner, W., Kempter, R., van Hemmen, L., and Wagner, H. (1996). A neuronal learning rule for sub-millisecond temporal coding. Nature 383, 76-78.

Gerstner, W., and Kistler, W. M. (2002). Spiking Neuron Models. Cambridge: Cambridge University Press.

Greuel, J. M., Luhmann, H. J., and Singer, W. (1988). Pharmacological induction of use-dependent receptive field modifications in the visual cortex. Science 242, 74-77.

Haas, J. S., Nowotny, T., and Abarbanel, H. D. I. (2006). Spike-timing-dependent plasticity of inhibitory synapses in the entorhinal cortex. J. Neurophysiol. 96, 3305-3313.

Harsanyi, K., and Friedlander, M. J. (1997). Transient synaptic potentiation in the visual cortex. I Cellular mechanisms. $J$ Neurophysiol. 77, 1269-1283.

Hebb, D. O. (1949). The Organization of Behavior. New York: John Wiley and Sons, 337.

Hein, A., Held, R., and Gower, E. C. (1970). Development and segmen- 
tation of visually-controlled movement by selective exposure during rearing. J. Comp. Physiol. Psychol. 73, 181-187.

Hein,A., Vital-Durand, F., Salinger,W., and Diamond, R. (1979). Eye movements initiate visual-motor development in the cat. Science 204, 1321-1322.

Heynen,A. J., and Bear, M. F. (2001). Longterm potentiation of thalamocortical transmission in the adult visual cortex in vivo. J. Neurosci. 21, 9801-9813.

Hirsch, H. V. B., and Spinelli, D. N. (1970). Visual experience modifies distribution of horizontally and vertically oriented receptive fields in cats. Science 168, 865-871.

Holmgren, C. D., and Zilberter, Y. (2007). Coincident spiking activity induces long-term changes in inhibition of neocortical pyramidal cells. J. Neurosci. 27, 8270-8277.

Iny, K., Heynen, A. J., Sklar, E., and Bear, M. F. (2006). Bidirectional modifications of visual acuity induced by monocular deprivation in juvenile and adult rats. J. Neurosci. 26, 7368-7374.

Jacob, V., Brasier, D. J., Erchova, I., Feldman, D., and Shulz, D. E. (2007). Spike timing-dependent synaptic depression in the in vivo barrel cortex of the rat. J. Neurosci. 27, 1271-1284.

Kampa, B. M., Letzkus, J. J., and Stuart, G. J. (2006). Requirement of dendritic calcium spikes for induction of spiketiming-dependent synaptic plasticity. J. Physiol. 574, 283-290.

Kara, P., Pezaris, J. S., Yurgenson, S., and Reid, R. C. (2002). The spatial receptive field of thalamic inputs to single cortical simple cells revealed by the interaction of visual and electrical stimulation. Proc. Natl. Acad. Sci. U.S.A. 99, 16261-16266.

Kasamatsu, T., Watabe, K., Heggelund, P., and Scholler, E. (1985). Plasticity in cat visual cortex restored by electrical stimulation of the locus coeruleus. Neurosci. Res. 2, 365-386.

Kelso, S. R., Ganong, A. H., and Brown, T. H. (1986). Hebbian synapses in hippocampus. Proc. Natl. Acad. Sci. U.S.A. 83, 5326-5330.

Kety,S. (1970). "Biogenic amines in the central nervous system: their possible roles in arousal, emotion and learning," in The Neurosciences: Second Study Program, ed.F.O.Schmitt (New York: Rockefeller University Press), 324-336.

Kirkwood, A., and Bear, M. F. (1994). Hebbian synapses in visual cortex. J. Neurosci. 14, 1634-1645.

Kirkwood, A., Dudek, S. M., Gold, J. T., Aizenman, C. D., and Bear, M. F. (1993). Common forms of synaptic plasticity in the hippocampus and neocortex in vitro. Science 260, 1518-1521.
Kirkwood, A, Lee, H. K., and Bear, M. F. (1995). Co-regulation of long-term potentiation and experience-dependent synaptic plasticity in visual cortex by age and experience. Nature 375, 328-331.

Kirkwood, A., Rioult, M. C., and Bear, M. F. (1996). Experience-dependent modification of synaptic plasticity in visual cortex. Nature 381,526-528.

Klopf, A. H. (1988). "Classical conditioning phenomena predicted by a drive-reinforcement model of neuronal function," in Neural Models of Plasticity, eds J. H. Byrne and W. O. Berry (New York: Academic Press), 104-132.

Kohonen, T. (1989). Self-Organization and Associative Memory. Berlin: Springer.

Komatsu, Y. (1994). Age-dependent longterm potentiation of inhibitory synaptic transmission in rat visual cortex. J. Neurosci. 14, 6488-6499.

Komatsu, Y. (1996). GABAB receptors, monoamine receptors, and postsynaptic inositol trisphosphate-induced $\mathrm{Ca} 2+$ release are involved in the induction of long-term potentiation at visual cortical inhibitory synapses. J. Neurosci. 16, 6342-6352.

Komatsu, Y., Fujii, K., Maeda, J., Sakaguchi, H., and Toyama, K. (1988). Long-term potentiation of synaptic transmission in kitten visual cortex. J. Neurophysiol. 59, 124-141.

Komatsu, Y., and Iwakiri, M. (1993). Longterm modification of inhibitory synaptic transmission in developing visual cortex. Neuroreport 4, 907-910.

Larkum, M. E., Kaiser, K. M. M., and Sakmann, B. (1999). Calcium electrogenesis in distal apical dendrites of layer 5 pyramidal cells at a critical frequency of back-propagating action potentials. Proc. Natl. Acad. Sci. U.S.A. 96, 14600-14604.

Letzkus, J. J., Kampa, B. M., and Stuart, G. J. (2006). Learning rules for spike timing-dependent plasticity depend on dendritic synapse location. J. Neurosci. 26, 10420-10429.

Levy, W. B. (1985). "Associative changes at the synapses: LTP in the hippocampus," in Synaptic Modification, Neuron Selectivity, and Nervous System Organization, eds W. B. Levy, J. A. Anderson, and S. Lehmkuhle (Hillsdale, NJ: Erlbaum), 5-34.

Levy, W. B., and Steward, O. (1983). Temporal contiguity requirements for long-term associative potentiation/depression in the hippocampus. Neuroscience 8, 791-797.

Lu, J. T., Li, C. Y., Zhao, J. P., Poo, M. M., and Zhang,X.H.(2007). Spike-timingdependent plasticity of neocortical excitatory synapses on inhibitory interneurons depends on target cell type. J. Neurosci. 27, 9711-9720.
Malenka, R. C. (1994). Synaptic plasticity in the hippocampus: LTP and LTD. Cell 78, 535-538.

Markram, H., Lübke, J., Frotscher, M., and Sakmann, B. (1997). Regulation of synaptic efficacy by coincidence of postsynaptic APs and EPSPs. Science 275, 213-215.

McLean, J., and Palmer, L. A. (1998). Plasticity of neuronal response properties in adult cat striate cortex. Vis. Neurosci. 15, 177-196.

Meliza, C. D., and Dan, Y. (2006). Receptive-field modification in rat visual cortex induced by paired visual stimulation and single-cell spiking. Neuron 49, 183-189.

Molyneaux, B. J., and Hasselmo, M. B. (2002). GABAB presynaptic inhibition has an in vivo time constant sufficiently rapid to allow modulation at theta frequency. J. Neurophysiol. 87, 1196-1205.

Mu, Y., and Poo, M.-M. (2006). Spike timing-dependent LTP/LTD mediates visual experience-dependent plasticity in a developing retinotectal system. Neuron 50, 115-125.

Mulkey, R. M., and Malenka, R. C. (1992). Mechanisms underlying induction of homosynaptic long-term depression in area CA1 of the hippocampus. Neuron 9, 967-975.

Nelson, S. B.(1991). Temporal interactions in the cat visual system. I. Orientationselective suppression in the visual cortex. J. Neurosci. 11, 344-356.

Oja,E. (1982).A simplified neuron model as a principal component analyzer. $J$. Math. Biol. 15, 267-273.

Pawlak, V., and Kerr, J. N. (2008). Dopamine receptor activation is required for corticostriatal spike-timing-dependent plasticity. J. Neurosci. 28, 2435-2446.

Pfister, J. P., and Gerstner, W. (2006). Triplets of spikes in a model of spike timing-dependent plasticity. $J$. Neurosci. 26, 9673-9682.

Rao, Y., and Daw, N. W. (2004). Layer variations of long-term depression in rat visual cortex. J. Neurophysiol. 92, 2652-2658.

Reiter, H. O., and Stryker, M. P. (1988). Neural plasticity without postsynaptic action potentials: less-active inputs become dominant when kitten visual cortical cells are pharmacologically inhibited. Proc. Natl. Acad. Sci. U.S.A. 85, 3623-3627.

Sejnowski, T. J. (1977). Statistical constraints on synaptic plasticity.J. Theor. Biol. 69, 387-389.

Sengpiel, F., Strawinski, P., and Bonhoeffer, T. (1999). Influence of experience on orientation maps in cat visual cortex. Nat. Neurosci. 2, 727-732.

Seol, G. H., Ziburkus, J., Huang, S., Song, L., Kim, I. T., Takamiya, K., Huganir, R.
L., Lee, H. K., and Kirkwood,A. (2007). Neuromodulators control the polarity of spike-timing-dependent synaptic plasticity. Neuron 55, 919-929.

Shulz, D., Debanne, D., and Frégnac, Y. (1993). Cortical convergence of ONand OFF-pathways, and functional adaptation of receptive field organization in cat area 17. Prog. Brain Res. 95, 191-205.

Shulz, D., and Frégnac, Y. (1992). Cellular analogs of visual cortical epigenesis: II. Plasticity of binocular integration. J. Neurosci. 12, 1301-1318.

Shulz, D.E., and Jacob,V. (2010). Spike timing dependent plasticity in the intact brain: counteracting spurious spike coincidences. Front. Neurosci. 4:137. doi: 10.3389/fnsyn.2010.00137.

Sjöström, P. J., and Häusser, M. (2006). A cooperative switch determines the sign of synaptic plasticity in distal dendrites of neocortical pyramidal neurons. Neuron 51, 227-238.

Sjöström, P. J., and Nelson, S. B. (2002). Spike timing, calcium signals and synaptic plasticity. Curr. Opin. Neurobiol. 12, 305-314.

Sjöström, P. J., Turrigiano, G. G., and Nelson, S. B. (2001). Rate, timing, and cooperativity jointly determine cortical synaptic plasticity. Neuron 32, 1149-1164.

Sjöström, P. J., Turrigiano, G. G., and Nelson, S.B.(2004). Endocannabinoiddependent neocortical layer-5 LTD in the absence of postsynaptic spiking. $J$. Neurophysiol. 92, 3338-3343.

Stent, G. (1973). A physiological mechanism for Hebb's postulate of learning. Proc. Natl. Acad. Sci. U.S.A. 70, 997-1001.

Stuart, G., Spruston, N., Sakmann, B., and Häusser, M. (1997). Action potential initiation and backpropagation in neurons of the mammalian CNS. Trends Neurosci. 20, 125-131.

Tanaka, S., Ribot, J., Imamura, K., and Tani, T. (2006). Orientation-restricted continuous visual exposure induces marked reorganization of orientation maps in early life. Neuroimage 30, 426-477.

Tao, H. W., Zhang, L. I., Engert, F., and Poo, M. (2001). Emergence of input specificity of lts during development of retinotectal connections in vivo. Neuron 31, 569-580.

Tsubokawa, T., Offermanns, S., Simon, M., and Kano, M. (2000). Calcium dependent persistent facilitation of spike backpropagation in the CA1 pyramidal neurons. J. Neurosci. 20, 4878-4884.

Turrigiano, G. G., and Nelson, S. B. (2000). Hebb and homeostasis in neuronal plasticity. 10, 358-364.

Tusa, R. J., Palmer, L. A., and Rosenquist, A. C. (1978). The retinotopic 
organization of area 17 (striate cortex) in the cat. J. Comp. Neurol. 177, 213-236.

Van den Burg, E., Engelmann, J., Bacelo, J., and Grant, K. (2007). Etomidate reduces initiation of backpropagating dend ritic action potentials: implications for sensory processing and synaptic plasticity during anesthesia. $J$. Neurophysiol. 97, 2373-2384.

van Rossum, M. C. W., Bi, Q., and Turrigiano, G. G. (2000). Stable Hebbian learning through spike-timing-dependent plasitcity. J. Neurosci. 20, 8812-8821.

Von der Malsburg, C. (1973). Selforganization of orientation sensitive cells in the striate cortex. Kybernetik 14, 85-100.
Wang, X., Merzenich, M. M., Sameshima, K., and Jenkins, W. M. (1995). Remodelling of hand representation in adult cortex determined by timing of tactile stimulation. Nature 378, 71-75.

Wiesel, T. N., and Hubel, D. H. (1963). Single-cell responses in striate cortex of kittens deprived of vision in one eye. J. Neurophysiol. 26, 1003-1017.

Wigström, H., and Gustafsson, B. (1985). On long-lasting potentiation in the hippocampus: a proposed mechanism for its dependence on coincident preand postsynaptic activity. Acta Physiol. Scand. 123, 519-522.

Wörgötter, F., and Eysel, U. T. (1987). Quantitative determination of orientational and directional components in the response of visual cortical cells to moving stimuli. Biol. Cybern. 57, 349-355.

Yao, H., and Dan, Y. (2001). Stimulus timing-dependent plasticity in cortical processing of orientation. Neuron 32, 315-323.

Young, J. M., Waleszczyk, W. J., Wang, C., Calford, M. B., Dreher, B., and Obermayer, K. (2007). Cortical reorganization consistent with spike timing- but not correlation-dependent plasticity. Nat. Neurosci. 10, 887-895.

Conflict of Interest Statement: The authors declare that the research was conducted in the absence of any commercial or financial relationships that could be construed as a potential conflict of interest.
Received: 19 May 2010; paper pending published: 29 June 2010; accepted: 28 October 2010; published online: 09 December 2010.

Citation: Frégnac Y, Pananceau M, René A, Huguet N, Marre O, Levy M and Shulz DE (2010) A re-examination of Hebbiancovariance rules and spike timing-dependent plasticity in cat visual cortex in vivo. Front. Syn. Neurosci. 2:147. doi: 10.3389/ fnsyn.2010.00147

Copyright (c) 2010 Frégnac, Pananceau, René, Huguet, Marre, Levyand Shulz. This is an open-access article subject to an exclusive license agreement between the authors and the Frontiers Research Foundation, which permits unrestricted use, distribution, and reproduction in any medium, provided the original authors and source are credited. 